WU-AP/60/96

\title{
Dynamics of Quiet Universes
}

\author{
Hiraku MUTOH, TOSHINARI HIRAIf and KeI-ICHI MAEDA円 \\ Department of Physics, Waseda University, Shinjuku-ku, Tokyo 169, Japan
}

\begin{abstract}
We study the stability of a contracting silent universe, which is a spacetime with irrotational dust and vanishing magnetic part of the Weyl tensor, $H_{a b}=0$. Two general relativistic backgrounds are analyzed; one is an attractor of silent universes, i.e., a locally Kasner spacetime, and the other is a particular class of inhomogeneous Szekeres solutions. In both cases their stabilities against perturbations with nonzero magnetic part depend on a contraction configuration; a spindle-like collapse is unstable, while a pancake-like collapse is still stable. We also find that a similar instability exists in spindle collapse for a Newtonian case. We conclude that $H_{a b}=0$ is not a generic ansatz even in general relativistic dust collapse.
\end{abstract}

Subject headings: cosmology: theory — galaxies: formation — relativity — largescale structure of universe

July, 1996

*e-mail: hirai@cfi.waseda.ac.jp

†e-mail: maeda@cfi.waseda.ac.jp 


\section{Introduction}

The recent observations of the large scale structure in the universe show that quite nonlinear wall-like structures or filament-like structures seem to exist. Such structures may give us important information about the structure formation process in the universe. It may be necessary to study nonlinear dynamics in order to know what kind of structure is formed on large scales.

In Newtonian gravity, the Zel'dovich approximation (Zel'dovich 1970), which describes well the evolution of inhomogeneities even in the weakly nonlinear regime, predicts a pancake collapse. The analysis of a homogeneous ellipsoid in an expanding universe also supports such a pancake collapse (White \& Silk 1979). How about for a very large scale such that general relativistic effects become important?

In general relativity, however, the analysis of nonlinear dynamics is quite difficult because the Einstein equations are highly nonlinear partial differential equations. It has been restricted to very simple cases such as the spherically symmetric Tolman-Bondi model (Tolman 1934; Bondi 1947). Recently the nonlinear dynamics of irrotational dust with vanishing magnetic part of the Weyl tensor $\left(H_{a b}=0\right)$ has been examined (Barnes \& Rowlingson 1989; Matarrese et al. 1993; Croudace et al. 1994; Bertschinger \& Jain 1994, Bruni et al. 1995a, b). Under such a condition, the system is described by nonlinear ordinary differential equations, which makes us able to analyze more generic situations. Such a system has no degree of freedom of gravitational waves, no information can transfer between the neighborhoods, and each fluid element evolves independently. Therefore such models were called silent universes by Matarrese et al. (1994). Bruni et al. (1995a) found attractor solutions in that system and showed that a generic gravitational contraction leads to a triaxial spindle-like configuration.

However the assumption of $H_{a b}=0$ is controversial. It may not be necessarily justified for generic cases. In fact, Matarrese et al. (1993) and Kojima (1994) pointed out, using a perturbative approach in an expanding Friedmann-Robertson-Walker (FRW) universe, that even if $H_{a b}=0$ in a linear regime, $H_{a b}$ will be generated during the nonlinear evolution of inhomogeneities and would affect the dynamics of the universe. This leads to another question, i.e., "Is the attractor in the silent universe still some attractor in more 
generic spacetimes?"

In this paper, we study perturbations of silent universes, taking into account the effect of non-vanishing $H_{a b}$. We shall call such a perturbed spacetime a quiet universe. Then the question above becomes "Will a quiet universe be silent ?" Analyzing stability of the attractor solution of silent universes (Kasner-type spacetime) and some particular class of Szekeres solutions (Szekeres 1975), we discuss whether or not the silent universe is generic in general relativity and what kind of configuration is more probable in the nonlinear regime of structure formation.

This paper is organized as follows. In $\S 2$, we write down the basic equations for the dynamics of irrotational dust with the introduction of dimensionless variables and we summarize the silent universes. In $\S 3$, the stability of silent universes against linear perturbations with non-vanishing $H_{a b}$ is analyzed. We consider two background spacetimes as unperturbed spacetimes: the Kasner-type universe and some Szekeres universes and show the dependence of stability on contraction configuration. We also discuss the stability of the Zel'dovich solution in Newtonian theory in $\S 4$. Our conclusion follows in $\S 5$.

We take $c=8 \pi G=1$, and the signature of $(-,+,+,+)$. Throughout this paper, Latin indices are used for coordinate components; $a, b, c, \cdots$ run from 0 to 3 and $i, j, k, \cdots$ are for the three spatial indices, while Greek indices are used for tetrad components; $\alpha, \beta, \gamma, \cdots$ run from 0 to 3 and $\lambda, \mu, \nu, \cdots$ are for spatial triad indices. The brackets () and [] denote symmetrization and anti-symmetrization for all indices.

\section{Dynamics of Irrotational Dust}

\subsection{Basic Equations for Irrotational Dust}

The irrotational dust fluid system with four-velocity $u^{a}$ is described by the following variables: the mass density $\rho$, the expansion scalar $\theta$, the shear tensor $\sigma_{a b}$ and electric and magnetic parts of the Weyl tensor $C_{a b c d} ; E_{a b}$ and $H_{a b}$. Those are defined as

$$
\begin{aligned}
\theta & =u_{; c}^{c}, \\
\sigma_{a b} & =u_{(a ; b)}-\frac{1}{3} h_{a b} \theta, \\
E_{a b} & =C_{a c b d} u^{c} u^{d},
\end{aligned}
$$




$$
H_{a b}={ }^{*} C_{a c b d} u^{c} u^{d}
$$

where $h_{a b} \equiv g_{a b}+u_{a} u_{b}$ is a projection tensor, and ${ }^{*} C_{a b c d} \equiv \frac{1}{2} \epsilon_{a b e f} C^{e f}$ is the dual of the Weyl tensor. The basic equations (Ellis 1971) are

$$
\begin{aligned}
& \dot{\theta}+\frac{1}{3} \theta^{2}+2 \sigma^{2}+\frac{1}{2} \rho=0, \\
& \dot{\rho}+\theta \rho=0 \\
& \dot{\sigma}_{a b}+\sigma_{a c} \sigma_{b}^{c}+\frac{2}{3} \theta \sigma_{a b}-\frac{2}{3} h_{a b} \sigma^{2}+E_{a b}=0, \\
& h^{a c} h^{b d} \dot{E}_{c d}+h^{c(a} \epsilon^{b) d e f} u_{d} H_{c e ; f}+h^{a b} \sigma^{c d} E_{c d}+\theta E^{a b}-3 E_{c}{ }^{(a} \sigma^{b) c} \\
& \quad=-\frac{1}{2} \rho \sigma^{a b}, \\
& h^{a c} h^{b d} \dot{H}_{c d}-h^{c(a} \epsilon^{b) d e f} u_{d} E_{c e ; f}+h^{a b} \sigma^{c d} H_{c d}+\theta H^{a b}-3 H_{c}{ }^{(a} \sigma^{b) c} \\
& \quad=0, \\
& \frac{2}{3} \theta^{; a}-\sigma_{; b}^{a b}=0, \\
& H_{a b}=-h_{a c} h_{b d} \sigma_{e}{ }^{(c} ; f^{d) g e f} u_{g}, \\
& h^{a c} h^{b d} E_{c b ; d}-\epsilon^{a b c d} u_{b} \sigma^{e}{ }_{c} H_{d e}=\frac{1}{3} h^{a b} \rho_{; b}, \\
& h^{a c} h^{b d} H_{c b ; d}+\epsilon^{a b c d} u_{b} \sigma_{c}^{e} E_{d e}=0,
\end{aligned}
$$

where, an overdot represents a directional derivative along the fluid worldlines, e.g., $\dot{T}_{a \cdots b}=$ $T_{a \cdots b ; c} u^{c}$.

We introduce the orthonormal tetrad $\left\{e_{(\alpha)}^{a}\right\}$ by

$$
e_{(0)}^{a}=u^{a}=(1,0,0,0), \quad e_{(\mu)}^{a}=\left(0, e_{(\mu)}^{i}\right),
$$

with

$$
e_{(\mu)}^{i} e_{i}^{(\nu)}=\delta_{\mu}^{\nu} .
$$

The spatial triad vectors $\left\{e_{(\alpha)}^{a}\right\}$ are chosen to be Fermi-propagated along $u^{a}$, i.e.,

$$
e_{a ; b}^{(\alpha)} u^{b}=0 .
$$

The metric is described by those tetrad vectors as,

$$
\begin{aligned}
d s^{2} & =g_{a b} d x^{a} d x^{b} \\
& =-d t^{2}+e_{i(\mu)} e_{j}^{(\mu)} d x^{i} d x^{j} .
\end{aligned}
$$


To write down the basic equations in terms of the tetrad components, we use the following relation for the covariant derivatives:

$$
\begin{aligned}
T_{\alpha \beta ; \gamma} & =T_{a b ; c} e_{(\alpha)}^{a} e_{(\beta)}^{b} e^{c}{ }_{(\gamma)} \\
& =\partial_{\gamma} T_{\alpha \beta}-\Gamma_{\gamma \alpha}^{\delta} T_{\delta \beta}-\Gamma_{\gamma \beta}^{\delta} T_{\alpha \delta},
\end{aligned}
$$

where $\Gamma_{\alpha \beta \gamma} \equiv e^{a}{ }_{(\alpha)} e_{(\gamma) a ; \beta} e^{b}{ }_{(\beta)}$ is the Ricci rotation coefficient and $\partial_{\alpha}$ represents the directional derivative with respect to $\mathbf{e}_{(\alpha)}$. The governing equations for the tetrad components then become the same ones for the coordinate components in Ellis (1971).

We also need the equations for the Ricci rotation coefficients $\Gamma_{\beta \gamma}^{\alpha}$ or the structure constants $\gamma_{\beta \gamma}^{\alpha}=\Gamma_{\beta \gamma}^{\alpha}-\Gamma_{\gamma \beta}^{\alpha}$ to close our system. Those equations are obtained from the Jacobi identities, i.e.,

$$
\partial_{[\delta} \gamma_{\gamma \beta]}^{\alpha}+\gamma_{[\delta \gamma}^{\epsilon} \gamma_{\beta] \epsilon}^{\alpha}=0 .
$$

From our choice of the tetrad, some of the components of $\Gamma_{\beta \gamma}^{\alpha}$ are known as

$$
\Gamma_{00}^{0}=0, \quad \Gamma_{00}^{\mu}=0, \quad \Gamma_{0 \nu}^{0}=0, \quad \Gamma_{0 \nu}^{\mu}=0
$$

and

$$
\gamma_{\nu 0}^{\mu}=\Gamma_{\nu 0}^{\mu}=\sigma_{\mu \nu}+\frac{1}{3} \delta_{\mu \nu} \theta .
$$

Next, we define dimensionless variables, $\Omega, \Sigma_{\mu \nu}, \mathcal{E}_{\mu \nu}, \mathcal{H}_{\mu \nu}, \mathcal{G}_{\mu \nu \rho}$ and $\mathcal{C}_{\mu \nu \rho}$ as,

$$
\begin{aligned}
& \rho=\frac{1}{3} \Omega \theta^{2}, \sigma_{\mu \nu}=\Sigma_{\mu \nu} \theta, E_{\mu \nu}=\mathcal{E}_{\mu \nu} \theta^{2}, H_{\mu \nu}=\mathcal{H}_{\mu \nu} \theta^{2}, \\
& \Gamma_{\mu \nu \rho}=\mathcal{G}_{\mu \nu \rho} \theta, \gamma_{\mu \nu \rho}=\mathcal{C}_{\mu \nu \rho} \theta
\end{aligned}
$$

and

$$
\Sigma^{2}=\frac{1}{2} \Sigma_{\rho \sigma} \Sigma^{\rho \sigma} .
$$

Using those variables, an attractor solution in silent universes becomes a fixed point in a "phase" space. Now the basic equations for irrotational dust fluid are

$$
\begin{aligned}
\theta^{\prime} & =\frac{1}{6}\left(2+\Omega+12 \Sigma^{2}\right) \theta, \\
\Omega^{\prime} & =\frac{1}{3}\left(1-\Omega-12 \Sigma^{2}\right) \Omega, \\
\Sigma_{\mu \nu}^{\prime} & =\frac{1}{6}\left(2-\Omega-12 \Sigma^{2}\right) \Sigma_{\mu \nu}+\Sigma_{\mu \rho} \Sigma_{\nu}^{\rho}-\frac{2}{3} \delta_{\mu \nu} \Sigma^{2}+\mathcal{E}_{\mu \nu},
\end{aligned}
$$




$$
\begin{aligned}
\mathcal{E}_{\mu \nu}^{\prime}= & \frac{1}{3}\left(1-\Omega-12 \Sigma^{2}\right) \mathcal{E}_{\mu \nu}+\delta_{\mu \nu} \mathcal{E}_{\rho \sigma} \Sigma^{\rho \sigma}-\frac{3}{2}\left(\mathcal{E}_{\mu \rho} \Sigma^{\rho}{ }_{\nu}+\Sigma_{\mu \rho} \mathcal{E}_{\nu}{ }_{\nu}\right)+\frac{1}{6} \Omega \Sigma_{\mu \nu} \\
& +\frac{1}{2 \theta}\left(\epsilon_{\nu \rho \sigma} \mathcal{H}_{\mu}{ }^{\rho ; \sigma}+\epsilon_{\mu \rho \sigma} \mathcal{H}_{\nu}{ }^{\rho ; \sigma}\right)+\frac{1}{\theta^{2}} \theta^{; \sigma}\left(\epsilon_{\nu \rho \sigma} \mathcal{H}_{\mu}{ }^{\rho}+\epsilon_{\mu \rho \sigma} \mathcal{H}^{\rho}{ }_{\nu}\right) \\
\mathcal{H}_{\mu \nu}^{\prime}= & \frac{1}{3}\left(1-\Omega-12 \Sigma^{2}\right) \mathcal{H}_{\mu \nu}+\delta_{\mu \nu} \mathcal{H}_{\rho \sigma} \Sigma^{\rho \sigma}-\frac{3}{2}\left(\mathcal{H}_{\mu \rho} \Sigma^{\rho}{ }_{\nu}+\Sigma_{\mu \rho} \mathcal{H}^{\rho}{ }_{\nu}\right) \\
& -\frac{1}{2 \theta}\left(\epsilon_{\nu \rho \sigma} \mathcal{E}_{\mu}{ }^{\rho ; \sigma}+\epsilon_{\mu \rho \sigma} \mathcal{E}_{\nu}{ }^{\rho ; \sigma}\right)-\frac{1}{\theta^{2}} \theta^{; \sigma}\left(\epsilon_{\nu \rho \sigma} \mathcal{E}_{\mu}^{\rho}+\epsilon_{\mu \rho \sigma} \mathcal{E}_{\nu}^{\rho}\right) \\
& \frac{\theta^{; \nu}}{\theta}\left(\Sigma_{\mu \nu}-\frac{2}{3} \delta_{\mu \nu}\right)+\Sigma_{\mu \nu}{ }^{; \nu}=0 \\
& \mathcal{H}_{\mu \nu}=-\frac{\epsilon_{\mu \rho \sigma}}{2}\left(\frac{1}{\theta^{2}} \theta^{; \sigma \sigma} \Sigma_{\nu}{ }^{\rho}+\frac{1}{\theta} \Sigma_{\nu}{ }_{\nu}{ }^{\rho ; \sigma}\right)-\frac{\epsilon_{\nu \rho \sigma}}{2}\left(\frac{1}{\theta^{2}} \theta^{; \sigma} \Sigma_{\mu}{ }^{\rho}+\frac{1}{\theta} \Sigma_{\mu}{ }^{\rho ; \sigma}\right) \\
& \mathcal{E}_{\mu \nu}{ }^{; \nu}+\frac{2}{\theta} \theta^{; \nu} \mathcal{E}_{\mu \nu}=\epsilon_{\mu \rho \sigma} \theta \Sigma^{\rho \nu} \mathcal{H}^{\sigma}{ }_{\nu}+\frac{1}{9}\left(\Omega_{; \mu}+\frac{2}{\theta} \theta_{; \mu} \Omega\right) \\
& \mathcal{H}_{\mu \nu}{ }_{\mu \nu}+\frac{2}{\theta} \theta^{; \nu} \mathcal{H}_{\mu \nu}=-\epsilon_{\mu \rho \sigma} \theta \Sigma^{\rho \nu} \mathcal{E}^{\sigma}{ }_{\nu},
\end{aligned}
$$

where prime $\left({ }^{\prime}\right)$ denotes the derivative with respect to $\tau \equiv-\int \theta d t$ and $\epsilon^{\mu \nu \rho}$ is the LeviCivita tensor. $\tau \sim \ln (\text { a proper volume })^{-1}$ plays the role of time in the contracting phase. The Jacobi identities are

$$
\partial_{[\delta} \mathcal{C}_{\gamma \beta]}^{\alpha}+\frac{1}{\theta} \mathcal{C}_{[\delta \gamma}^{\alpha} \partial_{\beta]} \theta+\theta \mathcal{C}_{[\delta \gamma}^{\epsilon} \mathcal{C}_{\beta] \epsilon}^{\alpha}=0
$$

which consist of 12 independent equations: 9 dynamical and 3 constraint equations. Hence we have now 26 evolution equations and 17 constraint equations.

\subsection{Silent Universes}

The silent universe is proposed by assuming that (i) the fluid is collisionless dust $(p=0)$, (ii) it has no vorticity $\left(\omega_{a b}=0\right)$, and (iii) the magnetic part of the Weyl tensor vanishes $\left(H_{a b}=0\right)$. Such an ansatz seems to be plausible because gravitational waves may have nothing to do with the structure formation process?

$\sigma_{a b}$ and $E_{a b}$ are diagonalized under this condition as shown by Barnes \& Rowlingson (1989). The two independent components are diagonal: $\sigma_{11}, \sigma_{22}$ and $E_{11}, E_{22}$. The metric is written as

$$
d s^{2}=-d t^{2}+\sum_{i=1}^{3} l_{i}^{2}(\boldsymbol{x}, t)\left(d x^{i}\right)^{2},
$$

\footnotetext{
${ }^{1}$ It turns out, however, that a contribution of the magnetic part of the Weyl tensor may not also be negligible even in the Newtonian limit (Bertinger and Hamilton 1994).
} 
and,

$$
\frac{i_{i}}{l_{i}}=\frac{1}{3} \theta+\sigma_{i i}
$$

where the $l_{i}$ 's represent the scale factors in the $i$-direction and $\sigma_{i i}$ is the $i$ - $i$ tetrad component of the shear tensor.

For the silent universes, the evolution equations are reduced to the following closed set of ordinary differential equations.

$$
\begin{aligned}
\theta^{\prime} & =\frac{1}{6}\left(2+\Omega+12 \Sigma^{2}\right) \theta \\
\Omega^{\prime} & =\frac{1}{3}\left(1-\Omega-12 \Sigma^{2}\right) \Omega \\
\Sigma_{+}^{\prime} & =\frac{1}{6}\left(2-\Omega-12 \Sigma^{2}\right) \Sigma_{+}-\frac{1}{\sqrt{3}}\left(\Sigma_{+}^{2}-\Sigma_{-}^{2}\right)+\mathcal{E}_{+}, \\
\Sigma_{-}^{\prime} & =\frac{1}{6}\left(2-\Omega-12 \Sigma^{2}\right) \Sigma_{-}+\frac{2}{\sqrt{3}} \Sigma_{+} \Sigma_{-}+\mathcal{E}_{-} \\
\mathcal{E}_{+}^{\prime} & =\frac{1}{3}\left(1-\Omega-12 \Sigma^{2}\right) \mathcal{E}_{+}+\sqrt{3}\left(\Sigma_{+} \mathcal{E}_{+}-\Sigma_{-} \mathcal{E}_{-}\right)+\frac{1}{6} \Sigma_{+} \Omega \\
\mathcal{E}_{-}^{\prime} & =\frac{1}{3}\left(1-\Omega-12 \Sigma^{2}\right) \mathcal{E}_{-}-\sqrt{3}\left(\Sigma_{+} \mathcal{E}_{-}+\Sigma_{-} \mathcal{E}_{+}\right)+\frac{1}{6} \Sigma_{-} \Omega
\end{aligned}
$$

whereft

$$
\begin{array}{ll}
\Sigma_{+}=\frac{\sqrt{3}}{2}\left(\Sigma_{11}+\Sigma_{22}\right), & \Sigma_{-}=\frac{1}{2}\left(\Sigma_{11}-\Sigma_{22}\right), \\
\mathcal{E}_{+}=\frac{\sqrt{3}}{2}\left(\mathcal{E}_{11}+\mathcal{E}_{22}\right), & \mathcal{E}_{-}=\frac{1}{2}\left(\mathcal{E}_{11}-\mathcal{E}_{22}\right),
\end{array}
$$

and

$$
\Sigma^{2}=\Sigma_{+}^{2}+\Sigma_{-}^{2}
$$

In the present case, the equations for $\mathcal{G}_{\alpha \beta \gamma}$, or $\mathcal{C}_{\alpha \beta \gamma}$ are decoupled. From the condition of $\dot{H}_{a b}=0$, we also find a new constraint equation. However, those are guaranteed to be satisfied from the dynamical and other constraint equations (Lesame et al. 1994).

Because of the absence of spatial derivatives in the evolution equations, each fluid element evolves without influence of the neighborhood. Hence it is called a silent universe. It includes not only homogeneous Bianchi I spacetimes, but also inhomogeneous spacetimes such as spherically symmetric Tolman-Bondi spacetimes or Szekeres solutions.

\footnotetext{
${ }^{2}$ This choice of \pm variables is different from the definition by Bruni et al (1995a), but is the same as that in usual discussion on Bianchi models.
} 
We see the evolution equations for 5 dynamical variables $\left\{\Omega, \Sigma_{ \pm}, \mathcal{E}_{ \pm}\right\}$are closed by themselves. Analyzing them, Bruni et al. (1995a) find attractor solutions in 5-dimensional "phase" space of $\left\{\Omega, \Sigma_{ \pm}, \mathcal{E}_{ \pm}\right\} . \theta$ is obtained from eq. (2.36) after solving those 5 variables.

The attractors in contracting spacetimes $(\theta<0)$ locally correspond to a family of vacuum Kasner spacetimes. The set of attractors is a circle in a 2-dimensional $\left\{\Sigma_{+}, \Sigma_{-}\right\}$ "phase" space (Fig. 1). Only three points on the circle of attractors correspond to a pancake collapse, i.e., two of the $l_{i}$ stay finite but the other one vanishes. The rest of the points of the attractors correspond to spindle collapse, i.e., two of $l_{i}$ 's vanish but the other one diverges. Bruni et al. (1995a) then concluded that contracting regions generally tend to be spindle-like configurations in silent universes.

\section{Quiet Universes: Magnetic Perturbations of Silent Universes}

In order to generalize the analysis by Bruni et al. (1995a), we consider linear perturbations with $H_{a b}$ of a silent universe. First we study the case in which the background solution is an attractor of contracting silent universes, i.e., the Kasner-type spacetimes in 33.1 . Secondly, as a background solution, we adopt a planar inhomogeneous Szekeres solution which approaches a particular attractor solution of silent universes ( $\$ 3.2)$. We denote the background quantities of the silent universe by a bar $\left(^{-}\right)$and the perturbed ones with tilde $(\sim)$, i.e.,

$$
\begin{aligned}
& \theta=\bar{\theta}(1+\tilde{\vartheta}), \quad \Omega=\bar{\Omega}+\tilde{\Omega}, \quad \Sigma_{\mu \nu}=\bar{\Sigma}_{\mu \nu}+\tilde{\Sigma}_{\mu \nu} \\
& \mathcal{E}_{\mu \nu}=\overline{\mathcal{E}}_{\mu \nu}+\tilde{\mathcal{E}}_{\mu \nu}, \quad \mathcal{H}_{\mu \nu}=\tilde{\mathcal{H}}_{\mu \nu} \\
& \mathcal{G}_{\mu \nu \rho}=\overline{\mathcal{G}}_{\mu \nu \rho}+\tilde{\mathcal{G}}_{\mu \nu \rho}, \quad \mathcal{C}_{\mu \nu \rho}=\overline{\mathcal{C}}_{\mu \nu \rho}+\tilde{\mathcal{C}}_{\mu \nu \rho}
\end{aligned}
$$

and

$$
\bar{\Sigma}^{2}=\frac{1}{2} \bar{\Sigma}_{\mu \nu} \bar{\Sigma}^{\mu \nu}
$$

Note that $\overline{\mathcal{H}}_{\mu \nu}=0$ because the background spacetime is a silent universe.

The background spacetime we analyze here has at least one homogeneous direction, which we choose to be $x$ (or 1 ). We then analyze only a single plane-wave mode of perturbations with a comoving wave number $\boldsymbol{k}=(k, 0,0)$. The spatial gradient $\partial_{1}$ is 
replaced as

$$
\hat{\partial}_{1} \equiv \frac{1}{\bar{\theta}} \partial_{1}=\frac{i k}{\bar{\theta} l_{1}} .
$$

In this case, the perturbation equations, which consist of 26 dynamical and 17 constraint equations, are divided into the following 4 groups, where we have introduced $\left\{\bar{\Sigma}_{ \pm}\right\}$, $\left\{\tilde{\Sigma}_{ \pm}\right\},\left\{\overline{\mathcal{E}}_{ \pm}\right\},\left\{\tilde{\mathcal{E}}_{ \pm}\right\}$, and $\left\{\tilde{\mathcal{H}}_{ \pm}\right\}$in the same way as Eq. (2.42).

(1) Group $1\left(\tilde{\vartheta}, \tilde{\Omega}, \tilde{\Sigma}_{ \pm}, \tilde{\mathcal{E}}_{ \pm}, \tilde{\mathcal{H}}_{23}, \tilde{\mathcal{C}}_{331}, \tilde{\mathcal{C}}_{221}\right)$ :

(1-1) 9 dynamical equations:

$$
\begin{aligned}
& \tilde{\vartheta}^{\prime}=\frac{1}{6}\left(2+\bar{\Omega}+12 \bar{\Sigma}^{2}\right) \tilde{\vartheta}+\frac{1}{6} \tilde{\Omega}+4\left(\bar{\Sigma}_{+} \tilde{\Sigma}_{+}+\bar{\Sigma}_{-} \tilde{\Sigma}_{-}\right), \\
& \tilde{\Omega}^{\prime}=\frac{1}{3}\left(1-\bar{\Omega}-12 \bar{\Sigma}^{2}\right) \bar{\Omega} \tilde{\vartheta}+\frac{1}{3}\left(1-2 \bar{\Omega}-12 \bar{\Sigma}^{2}\right) \tilde{\Omega} \\
& -8 \bar{\Omega}\left(\bar{\Sigma}_{+} \tilde{\Sigma}_{+}+\bar{\Sigma}_{-} \tilde{\Sigma}_{-}\right), \\
& \tilde{\Sigma}_{+}^{\prime}=\left[\frac{1}{6} \bar{\Sigma}_{+}\left(2-\bar{\Omega}-12 \bar{\Sigma}^{2}\right)-\frac{1}{\sqrt{3}}\left(\bar{\Sigma}_{+}^{2}-\bar{\Sigma}_{-}^{2}\right)+\overline{\mathcal{E}}_{+}\right] \tilde{\vartheta}-\frac{1}{6} \bar{\Sigma}_{+} \tilde{\Omega} \\
& +\frac{1}{6}\left(2-\bar{\Omega}-12 \bar{\Sigma}^{2}\right) \tilde{\Sigma}_{+}-4 \bar{\Sigma}_{+}\left(\bar{\Sigma}_{+} \tilde{\Sigma}_{+}+\bar{\Sigma}_{-} \tilde{\Sigma}_{-}\right)-\frac{2}{\sqrt{3}}\left(\bar{\Sigma}_{+} \tilde{\Sigma}_{+}-\bar{\Sigma}_{-} \tilde{\Sigma}_{-}\right) \\
& +\tilde{\mathcal{E}}_{+}, \\
& \tilde{\Sigma}_{-}^{\prime}=\left[\frac{1}{6} \bar{\Sigma}_{-}\left(2-\bar{\Omega}-12 \bar{\Sigma}^{2}\right)+\frac{2}{\sqrt{3}} \bar{\Sigma}_{+} \bar{\Sigma}_{-}+\overline{\mathcal{E}}_{-}\right] \tilde{\vartheta}-\frac{1}{6} \bar{\Sigma}_{-} \tilde{\Omega} \\
& +\frac{1}{6}\left(2-\bar{\Omega}-12 \bar{\Sigma}^{2}\right) \tilde{\Sigma}_{-}-4 \bar{\Sigma}_{-}\left(\bar{\Sigma}_{+} \tilde{\Sigma}_{+}+\bar{\Sigma}_{-} \tilde{\Sigma}_{-}\right)+\frac{2}{\sqrt{3}}\left(\bar{\Sigma}_{+} \tilde{\Sigma}_{-}+\bar{\Sigma}_{-} \tilde{\Sigma}_{+}\right) \\
& +\tilde{\mathcal{E}}_{-}, \\
& \tilde{\mathcal{E}}_{+}^{\prime}=\left[\frac{1}{3}\left(1-\bar{\Omega}-12 \bar{\Sigma}^{2}\right) \overline{\mathcal{E}}_{+}+\sqrt{3}\left(\bar{\Sigma}_{+} \overline{\mathcal{E}}_{+}-\bar{\Sigma}_{-} \overline{\mathcal{E}}_{-}\right)+\frac{1}{6} \bar{\Sigma}_{+} \bar{\Omega}\right] \tilde{\vartheta} \\
& +\frac{1}{6}\left(\bar{\Sigma}_{+}-2 \overline{\mathcal{E}}_{+}\right) \tilde{\Omega} \\
& +\frac{1}{3}\left(1-\bar{\Omega}-12 \bar{\Sigma}^{2}\right) \tilde{\mathcal{E}}_{+}+\sqrt{3}\left(\bar{\Sigma}_{+} \tilde{\mathcal{E}}_{+}-\bar{\Sigma}_{-} \tilde{\mathcal{E}}_{-}\right)+\frac{1}{6} \bar{\Omega} \tilde{\Sigma}_{+} \\
& -\left(8 \bar{\Sigma}_{+}-\sqrt{3}\right) \overline{\mathcal{E}}_{+} \tilde{\Sigma}_{+}-\left(8 \bar{\Sigma}_{-} \overline{\mathcal{E}}_{+}+\sqrt{3} \overline{\mathcal{E}}_{-}\right) \tilde{\Sigma}_{-}+\frac{\sqrt{3}}{2} \hat{\partial}_{1} \tilde{\mathcal{H}}_{23}, \\
& \tilde{\mathcal{E}}_{-}^{\prime}=\left[\frac{1}{3}\left(1-\bar{\Omega}-12 \bar{\Sigma}^{2}\right) \overline{\mathcal{E}}_{-}-\sqrt{3}\left(\bar{\Sigma}_{+} \overline{\mathcal{E}}_{-}+\bar{\Sigma}_{-} \overline{\mathcal{E}}_{+}\right)+\frac{1}{6} \bar{\Sigma}_{-} \bar{\Omega}\right] \tilde{\vartheta} \\
& +\frac{1}{6}\left(\bar{\Sigma}_{-}-2 \overline{\mathcal{E}}_{-}\right) \tilde{\Omega} \\
& +\frac{1}{3}\left(1-\bar{\Omega}-12 \bar{\Sigma}^{2}\right) \tilde{\mathcal{E}}_{-}-\sqrt{3}\left(\bar{\Sigma}_{+} \tilde{\mathcal{E}}_{-}+\bar{\Sigma}_{-} \tilde{\mathcal{E}}_{+}\right)+\frac{1}{6} \bar{\Omega} \tilde{\Sigma}_{-} \\
& -\left(8 \bar{\Sigma}_{+}+\sqrt{3}\right) \overline{\mathcal{E}}_{-} \tilde{\Sigma}_{+}-\left(8 \bar{\Sigma}_{-} \overline{\mathcal{E}}_{-}+\sqrt{3} \overline{\mathcal{E}}_{+}\right) \tilde{\Sigma}_{-}-\frac{1}{2} \hat{\partial}_{1} \tilde{\mathcal{H}}_{23}, \\
& \tilde{\mathcal{H}}_{23}^{\prime}=\left[\frac{1}{3}\left(1-\bar{\Omega}-12 \bar{\Sigma}^{2}\right)+\frac{\sqrt{3}}{2}\left(\bar{\Sigma}_{+}+\sqrt{3} \bar{\Sigma}_{-}\right)\right] \tilde{\mathcal{H}}_{23}+\left(\sqrt{3} \overline{\mathcal{E}}_{+}-\overline{\mathcal{E}}_{-}\right) \hat{\partial}_{1} \tilde{\vartheta}
\end{aligned}
$$




$$
\begin{aligned}
+ & \frac{1}{2} \hat{\partial}_{1}\left(\sqrt{3} \tilde{\mathcal{E}}_{+}-\tilde{\mathcal{E}}_{-}\right)-\overline{\mathcal{E}}_{-} \tilde{\mathcal{G}}_{221}+\frac{1}{2}\left(\sqrt{3} \overline{\mathcal{E}}_{+}+\overline{\mathcal{E}}_{-}\right) \tilde{\mathcal{G}}_{331}, \\
\tilde{\mathcal{C}}_{331}^{\prime}=- & {\left[\frac{1}{6} \bar{\Omega}+2 \bar{\Sigma}^{2}-\frac{1}{\sqrt{3}}\left(\bar{\Sigma}_{+}+\sqrt{3} \bar{\Sigma}_{-}\right)\right] \tilde{\mathcal{C}}_{331}+\frac{2}{\sqrt{3}} \hat{\partial}_{1} \tilde{\Sigma}_{+} } \\
& -\frac{1}{3}\left(1-2 \sqrt{3} \bar{\Sigma}_{+}\right) \hat{\partial}_{1} \tilde{\vartheta} \\
\tilde{\mathcal{C}}_{221}^{\prime}=- & -\left[\frac{1}{6} \bar{\Omega}+2 \bar{\Sigma}^{2}-\frac{1}{\sqrt{3}}\left(\bar{\Sigma}_{+}+\sqrt{3} \bar{\Sigma}_{-}\right)\right] \tilde{\mathcal{C}}_{221}-\frac{1}{\sqrt{3}} \hat{\partial}_{1}\left(\tilde{\Sigma}_{+}-\sqrt{3} \tilde{\Sigma}_{-}\right) \\
& -\left[\frac{1}{3}+\frac{1}{\sqrt{3}}\left(\bar{\Sigma}_{+}-\sqrt{3} \bar{\Sigma}_{-}\right)\right] \hat{\partial}_{1} \tilde{\vartheta},
\end{aligned}
$$

(1-2) 3 constraint equations:

$$
\begin{aligned}
& {\left[\left(\bar{\Sigma}_{+}+\sqrt{3} \bar{\Sigma}_{-}\right)-\frac{2}{\sqrt{3}}\right] \hat{\partial}_{1} \tilde{\vartheta}+\hat{\partial}_{1}\left(\tilde{\Sigma}_{+}+\sqrt{3} \tilde{\Sigma}_{-}\right)+\sqrt{3}\left(\sqrt{3} \bar{\Sigma}_{+}+\bar{\Sigma}_{-}\right) \tilde{\mathcal{C}}_{331}} \\
& \quad+2 \sqrt{3} \bar{\Sigma}_{-} \tilde{\mathcal{C}}_{221}=0 \\
& \tilde{\mathcal{H}}_{23}=\frac{1}{2}\left[\left(\sqrt{3} \bar{\Sigma}_{+}-\bar{\Sigma}_{-}\right) \hat{\partial}_{1} \tilde{\vartheta}+\hat{\partial}_{1}\left(\sqrt{3} \tilde{\Sigma}_{+}-\tilde{\Sigma}_{-}\right)+\left(\sqrt{3} \bar{\Sigma}_{+}+\bar{\Sigma}_{-}\right) \tilde{\mathcal{C}}_{331}\right. \\
& \left.\quad-2 \bar{\Sigma}_{-} \tilde{\mathcal{C}}_{221}\right], \\
& \hat{\partial}_{1}\left(\tilde{\mathcal{E}}_{+}+\sqrt{3} \tilde{\mathcal{E}}_{-}\right)+2 \sqrt{3} \overline{\mathcal{E}}_{-} \tilde{\mathcal{C}}_{221}+\sqrt{3}\left(\sqrt{3} \overline{\mathcal{E}}_{+}+\overline{\mathcal{E}}_{-}\right) \tilde{\mathcal{C}}_{331} \\
& \quad+2\left[\left(\overline{\mathcal{E}}_{+}+\sqrt{3} \overline{\mathcal{E}}_{-}\right)-\frac{1}{3 \sqrt{3}} \bar{\Omega}\right] \hat{\partial}_{1} \tilde{\vartheta} \\
& \quad=\sqrt{3}\left(\sqrt{3} \bar{\Sigma}_{+}-\bar{\Sigma}_{-}\right) \tilde{\mathcal{H}}_{23}+\frac{1}{3 \sqrt{3}} \hat{\partial}_{1} \tilde{\Omega} .
\end{aligned}
$$

(2) Group $2\left(\tilde{\Sigma}_{23}, \tilde{\mathcal{E}}_{23}, \tilde{\mathcal{H}}_{ \pm}, \tilde{\mathcal{C}}_{123}, \tilde{\mathcal{C}}_{231}, \tilde{\mathcal{C}}_{312}\right)$ :

(2-1) 7 dynamical equations:

$$
\begin{aligned}
\tilde{\Sigma}_{23}^{\prime}= & \frac{1}{6}\left[2-\bar{\Omega}-12 \bar{\Sigma}^{2}-2 \sqrt{3}\left(\bar{\Sigma}_{+}+\sqrt{3} \bar{\Sigma}_{-}\right)\right] \tilde{\Sigma}_{23}+\tilde{\mathcal{E}}_{23} \\
\tilde{\mathcal{E}}_{23}^{\prime}= & {\left[\frac{1}{3}\left(1-\bar{\Omega}-12 \bar{\Sigma}^{2}\right)+\frac{\sqrt{3}}{2}\left(\bar{\Sigma}_{+}+\sqrt{3} \bar{\Sigma}_{-}\right)\right] \tilde{\mathcal{E}}_{23} } \\
& \quad+\frac{1}{6}\left[\bar{\Omega}+3 \sqrt{3}\left(\overline{\mathcal{E}}_{+}+\sqrt{3} \overline{\mathcal{E}}_{-}\right)\right] \tilde{\Sigma}_{23}-\frac{1}{2} \hat{\partial}_{1}\left(\sqrt{3} \tilde{\mathcal{H}}_{+}-\tilde{\mathcal{H}}_{-}\right) \\
\tilde{\mathcal{H}}_{+}^{\prime}= & \frac{1}{3}\left(1-\bar{\Omega}-12 \bar{\Sigma}^{2}\right) \tilde{\mathcal{H}}_{+}+\sqrt{3}\left(\bar{\Sigma}_{+} \tilde{\mathcal{H}}_{+}-\bar{\Sigma}_{-} \tilde{\mathcal{H}}_{-}\right)-\frac{\sqrt{3}}{2} \hat{\partial}_{1} \tilde{\mathcal{E}}_{23} \\
& -\frac{\sqrt{3}}{2}\left[\left(\sqrt{3} \overline{\mathcal{E}}_{+}+\overline{\mathcal{E}}_{-}\right) \tilde{\mathcal{G}}_{123}+\left(\sqrt{3} \overline{\mathcal{E}}_{+}-\overline{\mathcal{E}}_{-}\right) \tilde{\mathcal{G}}_{312}\right] \\
\tilde{\mathcal{H}}_{-}^{\prime}= & \frac{1}{3}\left(1-\bar{\Omega}-12 \bar{\Sigma}^{2}\right) \tilde{\mathcal{H}}_{-}-\sqrt{3}\left(\bar{\Sigma}_{+} \tilde{\mathcal{H}}_{-}+\bar{\Sigma}_{-} \tilde{\mathcal{H}}_{+}\right)+\frac{1}{2} \hat{\partial}_{1} \tilde{\mathcal{E}}_{23} \\
& \quad-\frac{1}{2}\left(\sqrt{3} \overline{\mathcal{E}}_{+}+\overline{\mathcal{E}}_{-}\right) \tilde{\mathcal{G}}_{123}+\frac{1}{2}\left(\sqrt{3} \overline{\mathcal{E}}_{+}-\overline{\mathcal{E}}_{-}\right) \tilde{\mathcal{G}}_{312}-2 \overline{\mathcal{E}}_{-} \tilde{\mathcal{G}}_{231}, \\
\tilde{\mathcal{C}}_{123}^{\prime}= & -\left[\frac{1}{6} \bar{\Omega}+2 \bar{\Sigma}^{2}+\frac{2}{\sqrt{3}}\left(\bar{\Sigma}_{+}+\sqrt{3} \bar{\Sigma}_{-}\right)\right] \tilde{\mathcal{C}}_{123},
\end{aligned}
$$




$$
\begin{aligned}
& \tilde{\mathcal{C}}_{231}^{\prime}=-\left[\frac{1}{6} \bar{\Omega}+2 \bar{\Sigma}^{2}+\frac{2}{\sqrt{3}}\left(\bar{\Sigma}_{+}-\sqrt{3} \bar{\Sigma}_{-}\right)\right] \tilde{\mathcal{C}}_{231}-\hat{\partial}_{1} \tilde{\Sigma}_{23} \\
& \tilde{\mathcal{C}}_{312}^{\prime}=-\left(\frac{1}{6} \bar{\Omega}+2 \bar{\Sigma}^{2}-\frac{4}{\sqrt{3}} \bar{\Sigma}_{+}\right) \tilde{\mathcal{C}}_{312}+\hat{\partial}_{1} \tilde{\Sigma}_{23}
\end{aligned}
$$

(2-2) 4 constraint equations:

$$
\begin{aligned}
& \tilde{\mathcal{H}}_{+}=-\frac{\sqrt{3}}{2}\left[\hat{\partial}_{1} \tilde{\Sigma}_{23}+\bar{\Sigma}_{-} \tilde{\mathcal{C}}_{123}-\bar{\Sigma}_{-} \tilde{\mathcal{C}}_{231}+\sqrt{3} \bar{\Sigma}_{+} \tilde{\mathcal{C}}_{312}\right], \\
& \tilde{\mathcal{H}}_{-}=\frac{1}{2}\left[\hat{\partial}_{1} \tilde{\Sigma}_{23}-\left(\sqrt{3} \bar{\Sigma}_{+}+2 \bar{\Sigma}_{-}\right) \tilde{\mathcal{C}}_{123}+\left(\sqrt{3} \bar{\Sigma}_{+}-2 \bar{\Sigma}_{-}\right) \tilde{\mathcal{C}}_{231}\right. \\
& \left.\quad+\bar{\Sigma}_{-} \tilde{\mathcal{C}}_{312}\right], \\
& \hat{\partial}_{1}\left(\tilde{\mathcal{H}}_{+}+\sqrt{3} \tilde{\mathcal{H}}_{-}\right)=\sqrt{3}\left(\sqrt{3} \overline{\mathcal{E}}_{+}-\overline{\mathcal{E}}_{-}\right) \tilde{\Sigma}_{23}-\sqrt{3}\left(\sqrt{3} \bar{\Sigma}_{+}-\bar{\Sigma}_{-}\right) \tilde{\mathcal{E}}_{23}, \\
& \hat{\partial}_{1} \tilde{\mathcal{C}}_{123}=0
\end{aligned}
$$

(3) Group $3\left(\tilde{\Sigma}_{31}, \tilde{\mathcal{E}}_{31}, \tilde{\mathcal{H}}_{12}, \tilde{\mathcal{C}}_{223}, \tilde{\mathcal{C}}_{113}\right)$ :

(3-1) 5 dynamical equations:

$$
\begin{aligned}
\tilde{\Sigma}_{31}^{\prime}= & \frac{1}{6}\left[2-\bar{\Omega}-12 \bar{\Sigma}^{2}-2 \sqrt{3}\left(\bar{\Sigma}_{+}-\sqrt{3} \bar{\Sigma}_{-}\right)\right] \tilde{\Sigma}_{31}+\tilde{\mathcal{E}}_{31}, \\
\tilde{\mathcal{E}}_{31}^{\prime}= & {\left[\frac{1}{3}\left(1-\bar{\Omega}-12 \bar{\Sigma}^{2}\right)+\frac{\sqrt{3}}{2}\left(\bar{\Sigma}_{+}-\sqrt{3} \bar{\Sigma}_{-}\right)\right] \tilde{\mathcal{E}}_{31} } \\
& \quad+\frac{1}{6}\left[\bar{\Omega}+3 \sqrt{3}\left(\overline{\mathcal{E}}_{+}-\sqrt{3} \overline{\mathcal{E}}_{-}\right)\right] \tilde{\Sigma}_{31}-\frac{1}{2} \hat{\partial}_{1} \tilde{\mathcal{H}}_{12}, \\
\tilde{\mathcal{H}}_{12}^{\prime}= & \frac{1}{3}\left(1-\bar{\Omega}-12 \bar{\Sigma}^{2}-3 \sqrt{3} \bar{\Sigma}_{+}\right) \tilde{\mathcal{H}}_{12}-\frac{1}{2} \hat{\partial}_{1} \tilde{\mathcal{E}}_{31} \\
& \quad+\frac{1}{2}\left[\left(\sqrt{3} \overline{\mathcal{E}}_{+}+\overline{\mathcal{E}}_{-}\right) \tilde{\mathcal{G}}_{113}-\left(\sqrt{3} \overline{\mathcal{E}}_{+}-\overline{\mathcal{E}}_{-}\right) \tilde{\mathcal{G}}_{223}\right] \\
\tilde{\mathcal{C}}_{223}^{\prime}= & -\left(\frac{1}{6} \bar{\Omega}+2 \bar{\Sigma}^{2}+\frac{2}{\sqrt{3}} \bar{\Sigma}_{+}\right) \tilde{\mathcal{C}}_{223}, \\
\tilde{\mathcal{C}}_{113}^{\prime}= & -\left(\frac{1}{6} \bar{\Omega}+2 \bar{\Sigma}^{2}+\frac{2}{\sqrt{3}} \bar{\Sigma}_{+}\right) \tilde{\mathcal{C}}_{113}+\hat{\partial}_{1} \tilde{\Sigma}_{31},
\end{aligned}
$$

(3-2) 5 constraint equations:

$$
\begin{aligned}
& \hat{\partial}_{1} \tilde{\Sigma}_{31}-\left(\sqrt{3} \bar{\Sigma}_{+}+\bar{\Sigma}_{-}\right) \tilde{\mathcal{C}}_{113}-\left(\sqrt{3} \bar{\Sigma}_{+}-\bar{\Sigma}_{-}\right) \tilde{\mathcal{C}}_{223}=0 \\
& \tilde{\mathcal{H}}_{12}=-\frac{1}{2}\left[\hat{\partial}_{1} \tilde{\Sigma}_{31}+\left(\sqrt{3} \bar{\Sigma}_{+}-\bar{\Sigma}_{-}\right) \tilde{\mathcal{C}}_{223}-\left(\sqrt{3} \bar{\Sigma}_{+}+\bar{\Sigma}_{-}\right) \tilde{\mathcal{C}}_{113}\right] \\
& \hat{\partial}_{1} \tilde{\mathcal{E}}_{31}-\left(\sqrt{3} \overline{\mathcal{E}}_{+}-\overline{\mathcal{E}}_{-}\right) \tilde{\mathcal{C}}_{223}-\left(\sqrt{3} \overline{\mathcal{E}}_{+}+\overline{\mathcal{E}}_{-}\right) \tilde{\mathcal{C}}_{113}=2 \bar{\Sigma}_{-} \tilde{\mathcal{H}}_{12}, \\
& \hat{\partial}_{1} \tilde{\mathcal{H}}_{12}=-\left(\sqrt{3} \overline{\mathcal{E}}_{+}+\overline{\mathcal{E}}_{-}\right) \tilde{\Sigma}_{31}+\left(\sqrt{3} \bar{\Sigma}_{+}+\bar{\Sigma}_{-}\right) \tilde{\mathcal{E}}_{31}, \\
& \partial_{1} \tilde{\mathcal{C}}_{223}=0
\end{aligned}
$$


(4) Group $4\left(\tilde{\Sigma}_{12}, \tilde{\mathcal{E}}_{12}, \tilde{\mathcal{H}}_{31}, \tilde{\mathcal{C}}_{112}, \tilde{\mathcal{C}}_{332}\right)$ :

(4-1) 5 dynamical equations:

$$
\begin{aligned}
\tilde{\Sigma}_{12}^{\prime}= & \frac{1}{6}\left(2-\bar{\Omega}-12 \bar{\Sigma}^{2}+4 \sqrt{3} \bar{\Sigma}_{+}\right) \tilde{\Sigma}_{12}+\tilde{\mathcal{E}}_{12} \\
\tilde{\mathcal{E}}_{12}^{\prime}= & \frac{1}{3}\left(1-\bar{\Omega}-12 \bar{\Sigma}^{2}-3 \sqrt{3} \bar{\Sigma}_{+}\right) \tilde{\mathcal{E}}_{12}-\left(\sqrt{3} \overline{\mathcal{E}}_{+}-\frac{1}{6} \bar{\Omega}\right) \tilde{\Sigma}_{12} \\
& +\frac{1}{2} \hat{\partial}_{1} \tilde{\mathcal{H}}_{31}, \\
\tilde{\mathcal{H}}_{31}^{\prime}= & {\left[\frac{1}{3}\left(1-\bar{\Omega}-12 \bar{\Sigma}^{2}\right)+\frac{\sqrt{3}}{2}\left(\bar{\Sigma}_{+}-\sqrt{3} \bar{\Sigma}_{-}\right)\right] \tilde{\mathcal{H}}_{31}+\frac{1}{2} \hat{\partial}_{1} \tilde{\mathcal{E}}_{12} } \\
& \quad-\frac{1}{2}\left(\sqrt{3} \overline{\mathcal{E}}_{+}-\overline{\mathcal{E}}_{-}\right) \tilde{\mathcal{G}}_{332}-\overline{\mathcal{E}}_{-} \tilde{\mathcal{G}}_{112}, \\
\tilde{\mathcal{C}}_{112}^{\prime}= & -\left[\frac{1}{6} \bar{\Omega}+2 \bar{\Sigma}^{2}-\frac{1}{\sqrt{3}}\left(\bar{\Sigma}_{+}-\sqrt{3} \bar{\Sigma}_{-}\right)\right] \tilde{\mathcal{C}}_{112}+\hat{\partial}_{1} \tilde{\Sigma}_{12}, \\
\tilde{\mathcal{C}}_{332}^{\prime}= & -\left[\frac{1}{6} \bar{\Omega}+2 \bar{\Sigma}^{2}-\frac{1}{\sqrt{3}}\left(\bar{\Sigma}_{+}-\sqrt{3} \bar{\Sigma}_{-}\right)\right] \tilde{\mathcal{C}}_{332},
\end{aligned}
$$

$\underline{(4-2) 5 \text { constraint equations }}$

$$
\begin{aligned}
& \hat{\partial}_{1} \tilde{\Sigma}_{12}+\left(\sqrt{3} \bar{\Sigma}_{+}-\bar{\Sigma}_{-}\right) \tilde{\mathcal{C}}_{332}-2 \bar{\Sigma}_{-} \tilde{\mathcal{C}}_{112}=0, \\
& \tilde{\mathcal{H}}_{31}=\frac{1}{2}\left[\hat{\partial}_{1} \tilde{\Sigma}_{12}-2 \bar{\Sigma}_{-} \tilde{\mathcal{C}}_{112}-\left(\sqrt{3} \bar{\Sigma}_{+}-\bar{\Sigma}_{-}\right) \tilde{\mathcal{C}}_{332}\right], \\
& \partial_{1} \tilde{\mathcal{E}}_{12}+\left(\sqrt{3} \overline{\mathcal{E}}_{+}-\overline{\mathcal{E}}_{-}\right) \tilde{\mathcal{C}}_{332}-2 \overline{\mathcal{E}}_{-} \tilde{\mathcal{C}}_{112}=-\left(\sqrt{3} \bar{\Sigma}_{+}+\bar{\Sigma}_{-}\right) \tilde{\mathcal{H}}_{31}, \\
& \hat{\partial}_{1} \tilde{\mathcal{H}}_{31}=2 \overline{\mathcal{E}}_{-} \tilde{\Sigma}_{12}-2 \bar{\Sigma}_{-} \tilde{\mathcal{E}}_{12}, \\
& \hat{\partial}_{1} \tilde{\mathcal{C}}_{332}=0 .
\end{aligned}
$$

Since each group is decoupled, 6 (in Group 1) and 3 (in Group 2) dynamical variables remain free at the initial time. Although we have fixed our tetrad system in the background spacetime, we still have some freedoms for their choice in perturbed spacetime. The time is chosen as the proper time of a dust particle, while the spatial coordinates have been left to be free in perturbations. As for the physical dynamical degrees of freedom of perturbations, we must have scalar and tensor modes, which are coupled except for the FRW spacetime background. Notice that we do not have a vector mode because of our irrotational dust. Since our perturbations are inhomogeneous only in the 1-direction, the tensor modes are decoupled into two parts; one is coupled to scalar perturbations (called even parity mode) and the other is decoupled (called odd parity mode). The perturbations of Group 1 then consist of scalar and even parity tensor modes, which have 4 
dynamical degrees of freedom. The other 2 variables are left still to be free because of the freedom of coordinate transformation in 2- and 3- directions. The perturbations of Group 2 consist of 2 physically dynamical freedoms (odd parity tensor modes) and 1 freedom of coordinate transformation in 1-direction. There is no degree of freedom in Groups 3 and 4, i.e., no physical perturbations.

Initially, we will specify $\tilde{\Omega}, \Sigma_{+}, \tilde{\mathcal{E}}_{+}$and $\tilde{\mathcal{H}}_{23}$ in Group 1 (corresponding to 4 physical modes). We set $\tilde{\mathcal{C}}_{331}=\tilde{\mathcal{C}}_{221}=0$, using remaining gauge freedoms, and determine other variables from the constraint equations. Then we analyze stability against the perturbations with 4 physically independent initial variables, $\left(\tilde{\Omega}, \tilde{\Sigma}_{+}, \tilde{\mathcal{E}}_{+}, \tilde{\mathcal{H}}_{23}\right)=(1+i, 0,0,0)$, $(0,1+i, 0,0),(0,0,1+i, 0)$, and $(0,0,0,1+i)$. In Group 2, we determine the initial data from 2 physical variables in the same way as Group 1.

For the first case of our analysis (§3.1), the choice of $x$ direction and a single wave mode does not cause a loss of generality, because $x$ is not a special direction in the present problem and any linear perturbations can be expanded by Fourier series. As for the second case (§3.2), the perturbations in another homogeneous direction $(y)$ will have the same properties as those in the $x$ direction. However, for the perturbations in the direction of inhomogeneity $(z)$, we may not be able to define invariant perturbations because the background itself is inhomogeneous. Therefore we have not analyzed it here.

\subsection{Perturbations of the Attractor Solutions}

The attractor solutions of silent universes are homogeneous vacuum Kasner type spacetimes, which are characterized by a constant value of $\eta,(0 \leq \eta<2 \pi)$, as

$$
\begin{aligned}
\bar{\Omega} & =0, \quad \bar{\Sigma}^{2}=\frac{1}{3}, \\
\bar{\Sigma}_{+} & =\frac{1}{\sqrt{3}} \cos \eta, \quad \bar{\Sigma}_{-}=\frac{1}{\sqrt{3}} \sin \eta \\
\overline{\mathcal{E}}_{+} & =\frac{\sqrt{3}}{9}(2 \cos \eta-1)(1+\cos \eta), \\
\overline{\mathcal{E}}_{-} & =-\frac{\sqrt{3}}{9}(2 \cos \eta-1) \sin \eta,
\end{aligned}
$$

and

$$
\bar{\theta}=\frac{\theta_{*}}{1+\theta_{*}\left(t-t_{*}\right)}
$$


where $\theta_{*}$ is the expansion at an initial time $t=t_{*}\left(\tau=\tau_{*}\right) . \tau$ is given as

$$
\tau-\tau_{*}=-\ln \left[1+\theta_{*}\left(t-t_{*}\right)\right]
$$

We have analyzed the behaviors of perturbations for various background values $\left(\Sigma_{+}\right)$ and for several scales of the perturbations $\left(k^{-1}\right)$.

The typical behavior of the perturbations is shown in Fig. 2, in which we set the initial values of the perturbations to $\left(\tilde{\Omega}, \tilde{\Sigma}_{+}, \tilde{\mathcal{E}}_{+}, \tilde{\mathcal{H}}_{23}\right)=(1+i, 0,0,0)$. We also analyze the other 3 independent initial data in Group 1, and find similar results.

The evolution of perturbations is quite different depending on whether the background collapse is spindle-like or pancake-like. Three pancake points are $\left(\bar{\Sigma}_{+}, \bar{\Sigma}_{-}\right)=\left(\frac{1}{2 \sqrt{3}}, \pm \frac{1}{2}\right)$, and $\left(-\frac{1}{\sqrt{3}}, 0\right)$. In the case of a spindle-like collapse, the magnetic part of the Weyl tensor gets larger and larger, and eventually diverges. The "silence" is broken. Other variables, e.g., some components of shear and the electric part of Weyl tensor also diverge. This asymptotic behavior is qualitatively independent of the wave number $k$ and background parameter $\eta$. On the other hand, in the case of a pancake-like collapse, the perturbations do not grow for any wave number. In pancake collapse, we have also analyzed another two independent perturbation modes, i.e., Group 2 modes and confirmed that it is stable.

As a result, we conclude that against generic perturbations with $H_{a b}$, a pancake-like attractor is still stable, but a spindle-like attractor becomes unstable.

\subsection{Perturbations of the Szekeres Solutions}

In the above analysis, since the background spacetime is a homogeneous attractor solution, our result might not be generic. It is possible that the evolution of the perturbations in an inhomogeneous background is different from that in a homogeneous background. Therefore we consider the following particular class of Szekeres solutions (Szekeres 1975;

Goode, S. W., \& Wainwright, J., 1982) as an inhomogeneous background silent universe:

$$
d s^{2}=-d t^{2}+t^{4 / 3}\left\{d x^{2}+d y^{2}+\left[1-t^{-1} \beta(z)\right]^{2} d z^{2}\right\}
$$

where $\beta(z)$ represents an inhomogeneity in the $z$-direction. When $\beta(z)=0$, the spacetime is just a flat Friedmann-Robertson-Walker (FRW) universe. 
The dimensionless variables defined above for this solution are given as

$$
\begin{aligned}
\bar{\theta} & =\frac{2 t-\beta(z)}{t(t-\beta(z))}, \\
\bar{\Omega} & =1-3 \bar{\Sigma}_{+}^{2}, \\
\overline{\mathcal{E}}_{+} & =\frac{1}{\sqrt{3}} \bar{\Sigma}_{+}\left(1+\sqrt{3} \bar{\Sigma}_{+}\right), \\
\overline{\mathcal{E}}_{-} & =\bar{\Sigma}_{-}=0,
\end{aligned}
$$

where

$$
\bar{\Sigma}_{+}=\frac{\beta(z)}{\sqrt{3}[\beta(z)-2 t]} .
$$

$\tau$ is given as

$$
\tau-\tau_{*}=-\ln \frac{t(t-\beta(z))}{t_{*}\left(t_{*}-\beta(z)\right)} .
$$

There is no stationary point in the "phase" space (see Fig.1). In the case of contracting phase, when $t$ decreases, even if the Szekeres universe is located initially near the isotropic FRW point $\left(\bar{\Sigma}_{+}=0\right)$, such spacetimes fall into different attractors according to the sign of $\bar{\Sigma}_{+}$, i.e., when $-1 / \sqrt{3}<\bar{\Sigma}_{+}<0$, it falls into a pancake-like attractor $\left(\bar{\Sigma}_{+}=-1 / \sqrt{3}\right)$, while if $0<\bar{\Sigma}_{+}<1 / \sqrt{3}$, then it approaches a spindle-like attractor $\left(\bar{\Sigma}_{+}=1 / \sqrt{3}\right.$ ) (see Fig.1).

Since in the $z$ direction, the background Szekeres solution is inhomogeneous, we focus only on the perturbations in the $x, y$ directions. Here we analyze only a single plane-wave mode with a wave number $\boldsymbol{k}=(k, 0,0)$, as mentioned before.

The results are shown in Figs. 3, in which we set the initial values of the perturbations $\left(\tilde{\Omega}, \tilde{\Sigma}_{+}, \tilde{\mathcal{E}}_{+}, \tilde{\mathcal{H}}_{23}\right)=(1+i, 0,0,0)$. We also analyze the other 3 independent initial data in Group 1, and find similar results. For a spindle-like background $\left(\bar{\Sigma}_{+}>0\right)$, the perturbation of the magnetic part of the Weyl tensor grows infinitely and the "silence" is broken. The electric part and the shear also diverge. On the other hand, for a pancake-like background, the perturbations do not grow. The result depends only on the contraction configuration, i.e., the sign of $\bar{\Sigma}_{+}$. It does not depend on other factors such as the spatial scale of perturbations. In pancake collapse, we have again analyzed another two independent perturbation modes, i.e., Group 2 modes and confirmed that it is stable.

Similarly to the Kasner-type background case, the present analysis shows that a pancake-like collapse is stable while a spindle-like collapse is unstable. One can also 
find that an isotropic collapse (FRW) is unstable against perturbations with magnetic part $H_{a b}$ (what is called the tensor mode).

Then some questions may arise, i.e., "Where will the destabilized solution approach in the "phase space" after a nonlinear evolution ?" or "How will the configuration change?" Our analysis shows that the perturbations with any wave length eventually approach the homogeneous one $(k=0)$. Therefore, we may expect that the spacetime always evolves locally into some Bianchi IX spacetime with complex oscillations near the singularity as shown in Belinskii et al (1970, 1982).

\section{Newtonian Case: Stability of Zel'dovich solutions}

Finally, in order to understand our results better, we analyze the stability of a silent universe in Newtonian gravity. The one-dimensional Zel'dovich solution in Newtonian gravity is very similar to the Szekeres solution in general relativity as pointed out by Kasai (1993), and then it may be regarded as a silent universe. Adopting the Zel'dovich solution as a background solution, we analyze its perturbations.

In Newtonian gravity, we can describe the irrotational dust fluid dynamics similar to the case in general relativity except for the absence of the Maxwell-like equations of $E_{i j}$ and $H_{i j}$ in general relativity (Ellis 1971). The system with irrotational dust fluid is described by a velocity field $v^{i}$, mass density $\rho$, expansion scalar $\theta=v^{i}{ }_{, i}$, shear tensor

$$
\sigma_{i j}=v_{(i, j)}-\frac{1}{3} \delta_{i j} \theta
$$

and tidal force

$$
E_{i j}=\phi_{, i j}-\frac{1}{3} \delta_{i j} \phi_{, l}^{, l}
$$

where $\phi$ is Newtonian gravitational potential.

The evolution equations of $\rho, \theta$, or $\sigma_{i j}$ have exactly the same expressions in general relativity, but $E_{i j}$ is determined only by its constraint equations without its evolution equation. $H_{i j}$ does not appear. The equations for dimensionless variables, $\Omega, \Sigma_{i j}, \mathcal{E}_{i j}$, defined by the same equations as (2.22), are

$$
\begin{aligned}
\theta^{\prime} & =\frac{1}{6}\left(2+\Omega+12 \Sigma^{2}\right) \theta, \\
\Omega^{\prime} & =\frac{1}{3}\left(1-\Omega-12 \Sigma^{2}\right) \Omega,
\end{aligned}
$$




$$
\begin{aligned}
& \Sigma_{i j}^{\prime}=\frac{1}{6}\left(2-\Omega-12 \Sigma^{2}\right) \Sigma_{i j}+\Sigma_{i l} \Sigma_{j}^{l}-\frac{2}{3} \delta_{i j} \Sigma^{2}+\mathcal{E}_{i j}, \\
& \frac{\theta_{, j}}{\theta}\left(\Sigma^{i j}-\frac{2}{3} \delta^{i j}\right)+\Sigma_{, j}^{i j}=0, \\
& \epsilon^{i l m}\left(\Sigma_{j l, m}+\frac{1}{\theta} \theta_{, m} \Sigma_{j l}\right)+\epsilon_{j l m}\left(\Sigma^{i l, m}+\frac{1}{\theta} \theta^{, m} \Sigma^{i l}\right)=0, \\
& \mathcal{E}^{i j}{ }_{, j}+\frac{2}{\theta} \theta_{, j} \mathcal{E}^{i j}=\frac{1}{9}\left(\Omega^{, i}+\frac{2}{\theta} \theta^{, i} \Omega\right), \\
& \epsilon^{i l m}\left(\mathcal{E}_{j l, m}+\frac{2}{\theta} \theta_{, m} \mathcal{E}_{j l}\right)+\epsilon_{j l m}\left(\mathcal{E}^{i l, m}+\frac{2}{\theta} \theta^{, m} \mathcal{E}^{i l}\right)=0,
\end{aligned}
$$

where $\Sigma^{2}=\Sigma_{l m} \Sigma^{l m} / 2$.

The one-dimensional Zel'dovich solution is obtained by transforming from the Eulerian coordinates $\boldsymbol{r}=\left(r_{1}, r_{2}, r_{3}\right)$ to the Lagrangian coordinates $\boldsymbol{q}=\left(q_{1}, q_{2}, q_{3}\right)$;

$$
\begin{aligned}
& r_{1}=t^{2 / 3} q_{1}, \\
& r_{2}=t^{2 / 3} q_{2}, \\
& r_{3}=t^{2 / 3}\left[q_{3}-D(t) S\left(q_{3}\right)\right],
\end{aligned}
$$

where $S\left(q_{3}\right)$ is an arbitrary function of $q_{3}$. Since we are interested in the contraction phase, we choose $D(t)=t^{-1}$ as $t$ decreases. This choice is different from the usual Zel'dovich solution, in which the growing mode of linear perturbation theory in an expanding universe is chosen $\left(D(t)=t^{2 / 3}\right.$ in the case of Einstein-de Sitter model). Although the perturbation of the Zel'dovich solution was already studied by Bildhauer et al. (1992), we reanalyze it here because our choice of $D(t)$ is different from theirs.

Transmission of information will take place due to the Poisson equation, so non-locality exists in general as is well known in Newtonian gravity. However, the Zel'dovich solution can be called a silent universe, because once an initial condition is set, that is, $S\left(q_{3}\right)$ is given, each fluid element evolves independently of other neighbors.

Therefore, the perturbation analysis can be done in the same way as in 83.2 . In fact the background variables are given by the same solutions as eqs. (3.54) (3.58) with replacement of $\beta(z)$ with $S\left(q_{3}\right)$. We analyze only perturbations in the $x$ direction with a wave number $\boldsymbol{k}=(k, 0,0)$. The perturbation equations are

$$
\tilde{\vartheta}^{\prime}=\frac{1}{6}\left(2+\bar{\Omega}+12 \bar{\Sigma}_{+}^{2}\right) \tilde{\vartheta}+\frac{1}{6} \tilde{\Omega}+4 \bar{\Sigma}_{+} \tilde{\Sigma}_{+}
$$




$$
\begin{aligned}
& \tilde{\Omega}^{\prime}= \frac{1}{3}\left(1-\bar{\Omega}-12 \bar{\Sigma}_{+}^{2}\right) \bar{\Omega} \tilde{\vartheta}+\frac{1}{3}\left(1-2 \bar{\Omega}-12 \bar{\Sigma}_{+}^{2}\right) \tilde{\Omega} \\
&-8 \bar{\Omega} \bar{\Sigma}_{+} \tilde{\Sigma}_{+}, \\
& \tilde{\Sigma}_{+}^{\prime}= {\left[\overline{\mathcal{E}}_{+}+\frac{1}{6}\left(2-\bar{\Omega}-2 \sqrt{3}\left(1+2 \sqrt{3} \bar{\Sigma}_{+}\right) \bar{\Sigma}_{+}\right) \bar{\Sigma}_{+}\right] \tilde{\vartheta} } \\
&-\frac{1}{6} \bar{\Sigma}_{+} \tilde{\Omega}+\tilde{\mathcal{E}}_{+}+\frac{1}{6}\left[2-\bar{\Omega}-4 \sqrt{3} \bar{\Sigma}_{+}\left(1+3 \sqrt{3} \bar{\Sigma}_{+}\right)\right] \tilde{\Sigma}_{+}, \\
& \tilde{\Sigma}_{-}^{\prime}= \tilde{\mathcal{E}}_{-}+\frac{1}{6}\left[2-\bar{\Omega}+4 \sqrt{3} \bar{\Sigma}_{+}\left(1-\sqrt{3} \bar{\Sigma}_{+}\right)\right] \tilde{\Sigma}_{-}, \\
&\left(\sqrt{3} \bar{\Sigma}_{+}-2\right) \tilde{\vartheta}_{, 1}+\sqrt{3}\left(\tilde{\Sigma}_{+}+\sqrt{3} \tilde{\Sigma}_{-}\right)_{, 1}=0, \\
& 3 \sqrt{3}\left(\tilde{\mathcal{E}}_{+}+\sqrt{3} \tilde{\mathcal{E}}_{-}\right)_{, 1}+2\left(3 \sqrt{3} \overline{\mathcal{E}}_{+}-\bar{\Omega}\right) \tilde{\vartheta}_{, 1}=\tilde{\Omega}_{, 1}, \\
& \sqrt{3} \bar{\Sigma}_{+} \tilde{\vartheta}_{, 1}+\left(\sqrt{3} \tilde{\Sigma}_{+}-\tilde{\Sigma}_{-}\right)_{, 1}=0, \\
& 2 \sqrt{3} \overline{\mathcal{E}}_{+} \tilde{\vartheta}_{, 1}+\left(\sqrt{3} \tilde{\mathcal{E}}_{+}-\tilde{\mathcal{E}}_{-}\right)_{, 1}=0, \\
& \tilde{\Sigma}_{12,1}=\tilde{\Sigma}_{13,1}=\tilde{\Sigma}_{23,1}=\tilde{\mathcal{E}}_{12,1}=\tilde{\mathcal{E}}_{13,1}=\tilde{\mathcal{E}}_{23,1}=0 .
\end{aligned}
$$

We have 6 variables with 4 constraint equations. Then the degree of freedom is two, which correspond to scalar perturbation modes. We find that the perturbations of $\tilde{\Sigma}_{i j}$ and $\tilde{\mathcal{E}}_{i j}$ behave very similarly to those in the case of the Szekeres background in general relativity. For a spindle-like collapse, the perturbations diverge, while for a pancake-like background, the perturbations decay (see Fig. 4, in which we set the initial values of the perturbations $\left(\tilde{\Omega}, \tilde{\mathcal{E}}_{+}\right)=(1+i, 0)$. We also analyze another set of independent initial data $\left(\tilde{\Omega}, \tilde{\mathcal{E}}_{+}\right)=(0,1+i)$, and find similar results). This result is consistent with the preference

of pancake collapse in Newtonian theory. The results suggest that the instability of a spindle collapse shown in $\oint 3$ is not due to an extreme situation near a singularity in general relativity.

\section{Conclusion}

We have studied the dynamics of a quiet universe, i.e., the perturbations of a silent universe with non-zero $H_{a b}$. The analysis of the silent universe by Bruni et al. (1995a) shows that a gravitational collapse prefers a spindle-like configuration. However, to consider a more generic case, we have analyzed perturbations with non-vanishing $H_{a b}$ for two general relativistic background solutions: (i) the attractors of the silent universe and (ii) a particular class of Szekeres solutions. In both cases, the behaviors of perturbations are 
qualitatively the same. The stability clearly depends on the configuration of a contracting background spacetime, i.e., either a pancake-like or a spindle-like collapse. In the case of a spindle-like collapse, the perturbations diverge and the silence is broken. The spacetime locally approaches the most generic homogeneous spacetime, that is, the Bianchi IX universe. On the other hand, the perturbations do not grow in the case of a pancake-like collapse. We conclude that even in general relativity, taking into account the effect of $H_{a b}$, a spindle collapse is destabilized.

This result agrees with the fact that a Newtonian collapse prefers a pancake-like configuration. Our Newtonian analysis of the stability of the Zel'dovich solution also supports this result. To know the relation between general relativistic perturbations with nonvanishing magnetic part and the Newtonian ones, we study the behavior of the magnetic part in the relativistic equation (2.27) (see Hui 1995). We find that the terms with the magnetic part diverge for a spindle collapse but keep finite for a pancake collapse. This suggests that the instability for a spindle collapse in Newtonian theory may also be caused by the magnetic part which is responsible for transmission of gravitational information. We conclude that an ansatz of silence $\left(H_{a b}=0\right)$ is no longer valid in generic gravitational contraction.

We would like to thank P. Haines and Y. Kojima for useful discussions. This work was supported partially by the Grant-in-Aid for Scientific Research Fund of the Ministry of Education, Science and Culture (No. 06302021 and No. 06640412), and by the Waseda University Grant for Special Research Projects. 


\section{References}

Barnes, A., \& Rowlingson, R. R. 1989, Class. Quantum Gravity, 6, 949

Belinskii, V. A., Khalatonikov, I. M., \& Lifshitz, E. M. 1970, Adv. Phys., 19, 525

—. 1982,Adv. Phys., 31, 639

Bertschinger, E., \& Jain, B. 1994, ApJ, 431, 486

Bertschinger, E. \& Hamilton, A. J. S. 1994, ApJ, 435, 1

Bildhauer, S., Aso, O., Kasai, M., \& Futamase,T. 1991. Mon. Not. R. astr. Soc. 252, 132

Bondi, H. 1947, MNRAS, 107, 410

Bruni, M., Matarrese, S., \& Pantano, O. 1995a, ApJ, 445, 958

—. 1995b, Phys. Rev. Lett., 74, 1916

Croudace, K. M., Parry, J., Salopek, D. S., \& Stewart, J. M. 1994, ApJ, 431, 22

Ellis, G. F. R. 1971, in General Relativity and Cosmology, ed. Sachs, R. K. (Academic, London), 104

Goode, S. W., \& Wainwright, J. 1982, Phys. Rev. D, 26, 3315

Hui, L., \& Bertschinger, E. 1995, ApJ, submitted

Kojima, Y. 1994, Phys. Rev. D, 50, 6110

Lesame, W. M., Dunsby, P. K. S., \& Ellis, G. F. R. 1995, Phys. Rev. D., 52, 3406

Matarrese, S., Pantano, O., \& Saez, D. 1993, Phys. Rev. D, 47, 1311

Matarrese, S., Pantano, O., \& Saez, D. 1994, Phys. Rev. Lett., 72, 320

Szekeres, P. 1975, Comm. Math. Phys., 41, 55

Tolman, R. C., 1934, Proc. Natl. Acad. Sci., 20, 169

White, S. D. M., \& Silk, J. 1979, ApJ, 231, 1

Zel'dovich, Ya. B. 1970, A \& A, 5, 84 


\section{Figure Captions}

Fig. 1: A set of attractors of silent universes in the $\left\{\Sigma_{+}, \Sigma_{-}\right\}$plane; three dots represent pancake-like attractors. The rest points on the circle correspond to spindle-like attractors. The line of $\Sigma_{-}=0\left(-1 / \sqrt{3}<\Sigma_{+}<1 / \sqrt{3}\right)$ shows a particular class of Szekeres solutions adopted as the background solution in 3.2 .

Fig. 2: The evolution of perturbations for the attractor of silent universes. The initial values of the perturbations are chosen as $\left(\tilde{\Omega}, \tilde{\Sigma}_{+}, \tilde{\mathcal{E}}_{+}, \tilde{\mathcal{H}}_{23}\right)=(1+i, 0,0,0)$. We set $\tilde{\mathcal{C}}_{221}=\tilde{\mathcal{C}}_{331}=0$ and other initial values are fixed by the constraint equations. Figs. 2(a) and 2(b) are in the case of pancake background $\left(\bar{\Sigma}_{+}=-1 / \sqrt{3}\right)$. Figs $2(\mathrm{c}), 2(\mathrm{~d})$ and $2(\mathrm{e}), 2(\mathrm{f})$ are in the case of spindle background $\left(\bar{\Sigma}_{+}=-1 /(4 \sqrt{3})\right.$ and $1 / \sqrt{3}$, respectively). The solid and dashed lines show the cases of the wave number $k / l_{1 *} \theta_{*}=10$ and $=1$, respectively.

Fig. 3: Fig. 3: The evolution of perturbations for a particular class of Szekeres solutions. The initial values of the perturbations are chosen as $\left(\tilde{\Omega}, \tilde{\Sigma}_{+}, \tilde{\mathcal{E}}_{+}, \tilde{\mathcal{H}}_{23}\right)=(1+i, 0,0,0)$. We set $\tilde{\mathcal{C}}_{221}=\tilde{\mathcal{C}}_{331}=0$ and other initial values are fixed by the constraint equations. Figs. 3(a) and 3(b) are in the case of pancake background $\left(\bar{\Sigma}_{+}=-1 /(4 \sqrt{3})\right)$. Figs 3(c) and 3(d) are in the case of spindle background $\left(\bar{\Sigma}_{+}=1 /(4 \sqrt{3})\right)$. The solid and dashed lines show the cases of the wave number $k / l_{1 *} \theta_{*}=10$ and $=1$, respectively. Figs. $3(\mathrm{e})$ and $3(\mathrm{f})$ are in the case of isotropic background $\left(\bar{\Sigma}_{+}=0\right)$. As for the isotropic(FRW) background, as was well known, the scalar perturbations are decoupled from the tensor modes. Hence, although our perturbation with the initial value $\left(\tilde{\Omega}, \tilde{\Sigma}_{+}, \tilde{\mathcal{E}}_{+}, \tilde{\mathcal{H}}_{23}\right)=(1+i, 0,0,0)$ diverges and the FRW spacetime is unstable against this perturbation(Fig. $3(\mathrm{e})), \tilde{\mathcal{H}}_{23}$ remains zero because it is a scalar mode. As for initial perturbations with $\tilde{\mathcal{H}}_{23} \neq 0, \tilde{\mathcal{H}}_{23}$ diverges and then the FRW spacetime is unstable against this tensor perturbation as well(Fig. 3(f)). Fig. 3(e) has just a solid line because the perturbation equations for the scalar mode of isotropic dust universe do not involve the wave number.

Fig. 4: The evolution of perturbations for the Zel'dovich solutions. The initial values of the perturbations are chosen as $\left(\tilde{\Omega}, \tilde{\mathcal{E}}_{+}\right)=(1+i, 0)$. Other initial values are fixed by the constraint equations. Fig. 4(a) is in the case of a pancake background $\left(\bar{\Sigma}_{+}=-1 /(4 \sqrt{3})\right)$, while Fig. 4(b) is in the case of a spindle background $\left(\bar{\Sigma}_{+}=1 /(4 \sqrt{3})\right)$. Figs. 4 are drawn with just a solid line because the perturbation equations do not involve the wave number. 


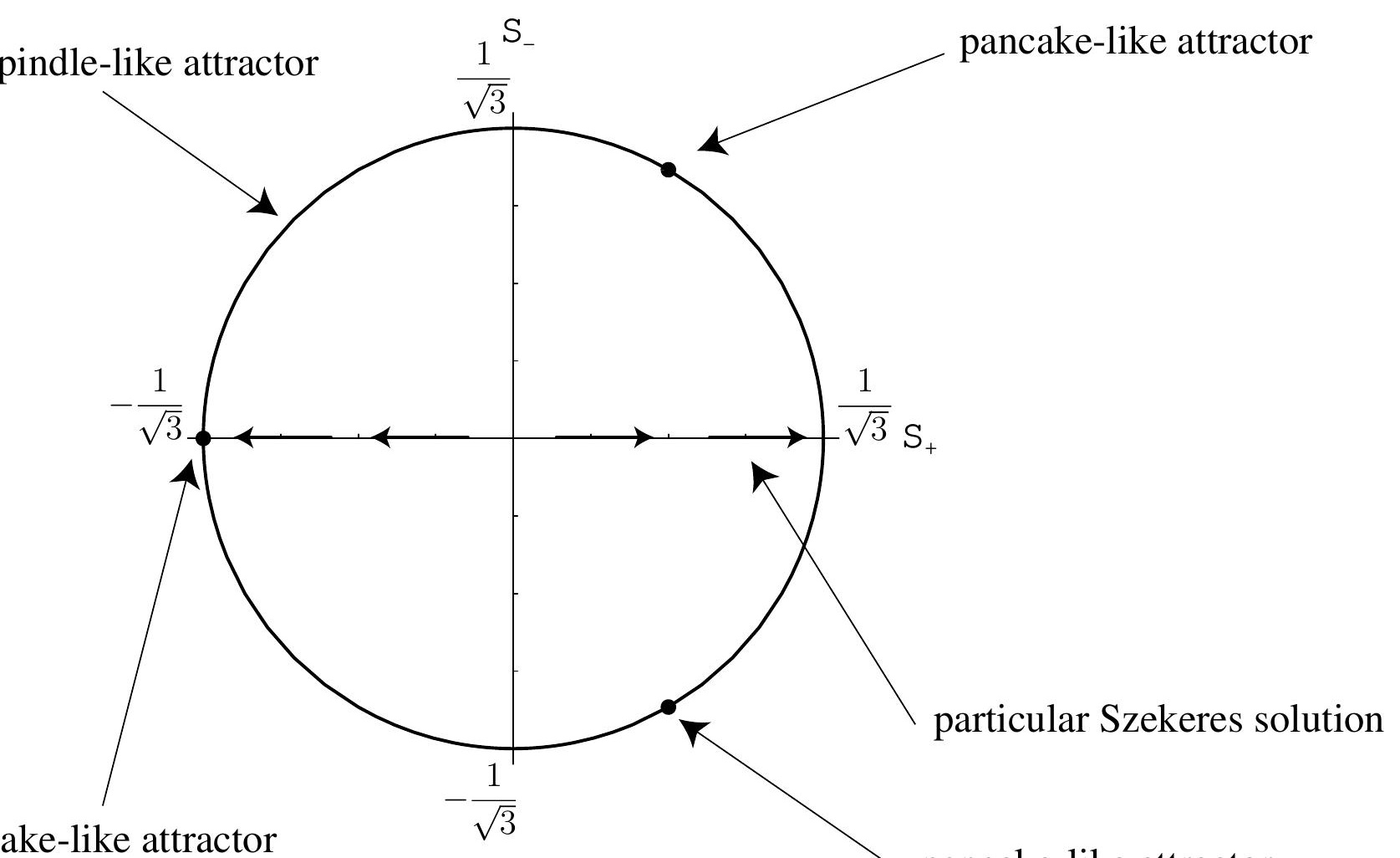




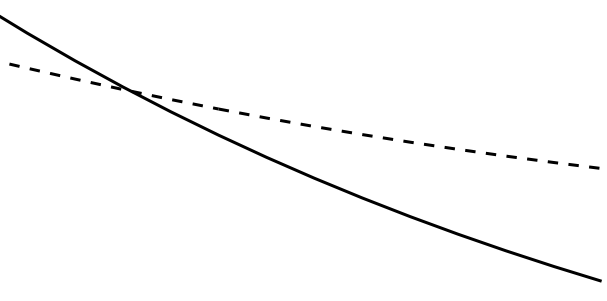




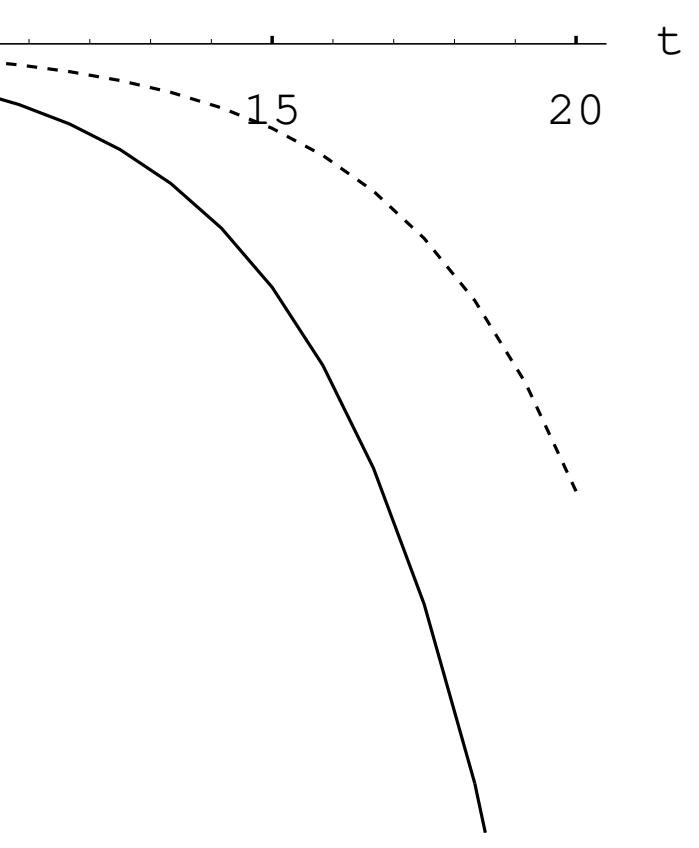




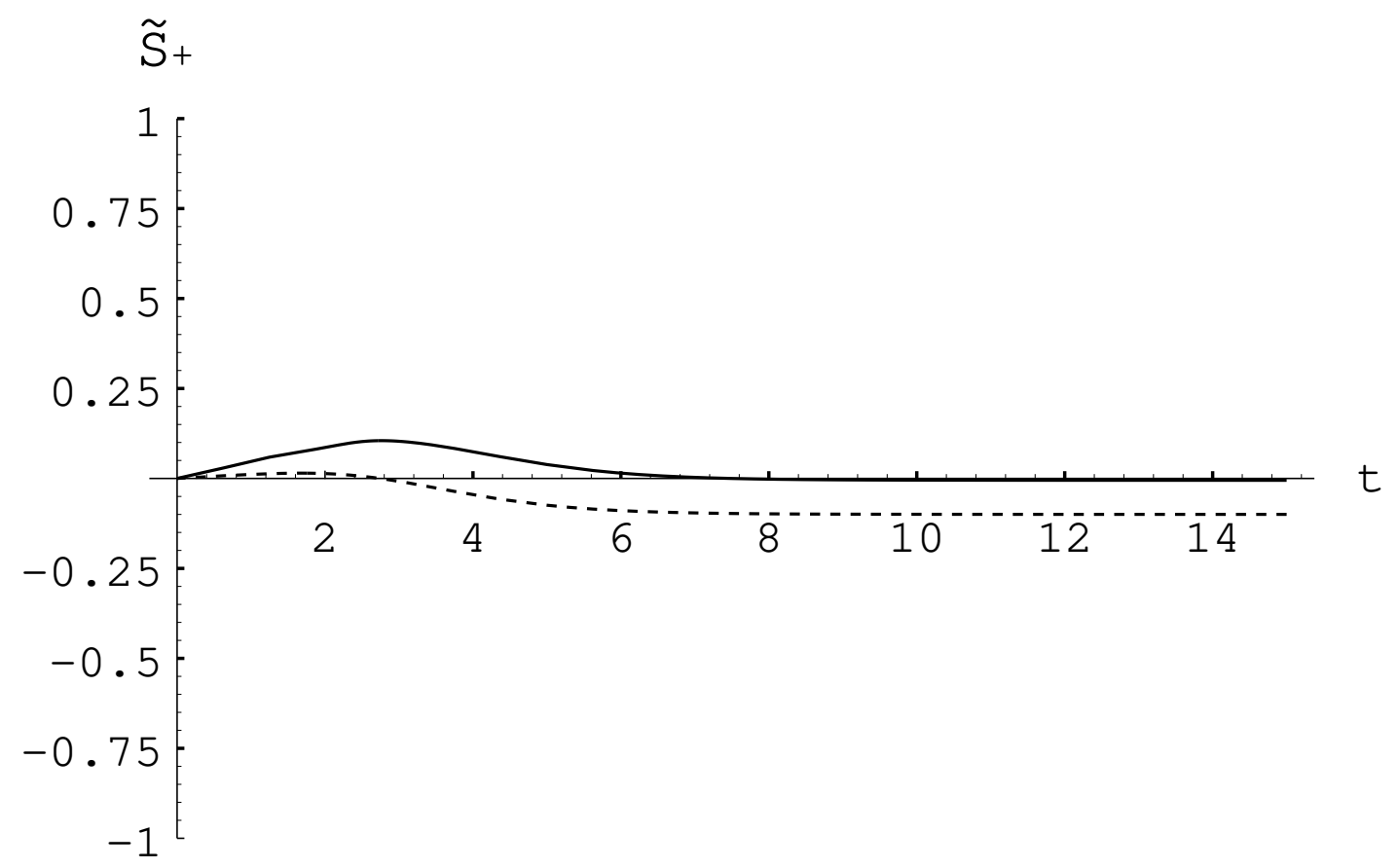

Fig. 3(a)

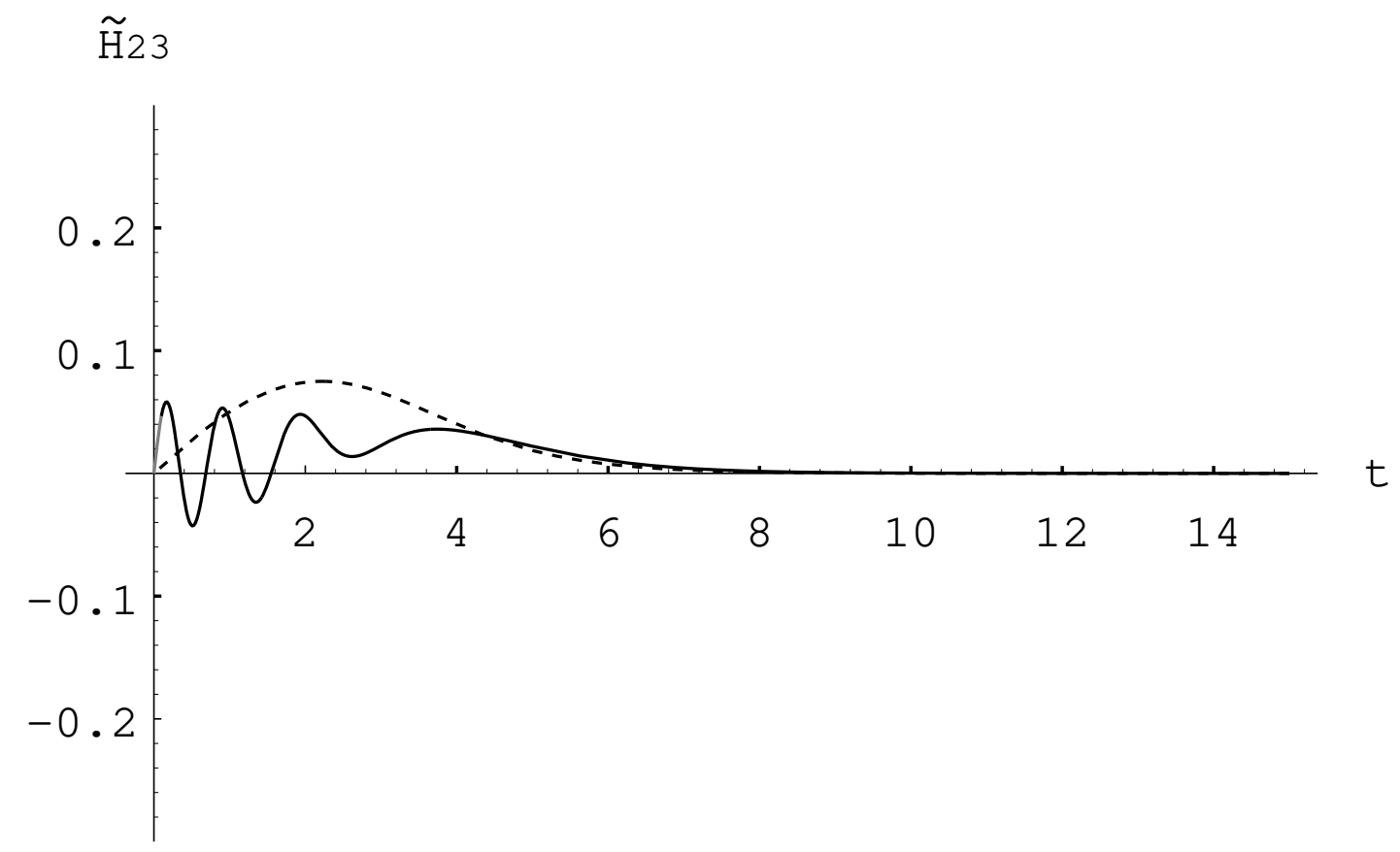

Fig. 3(b) 


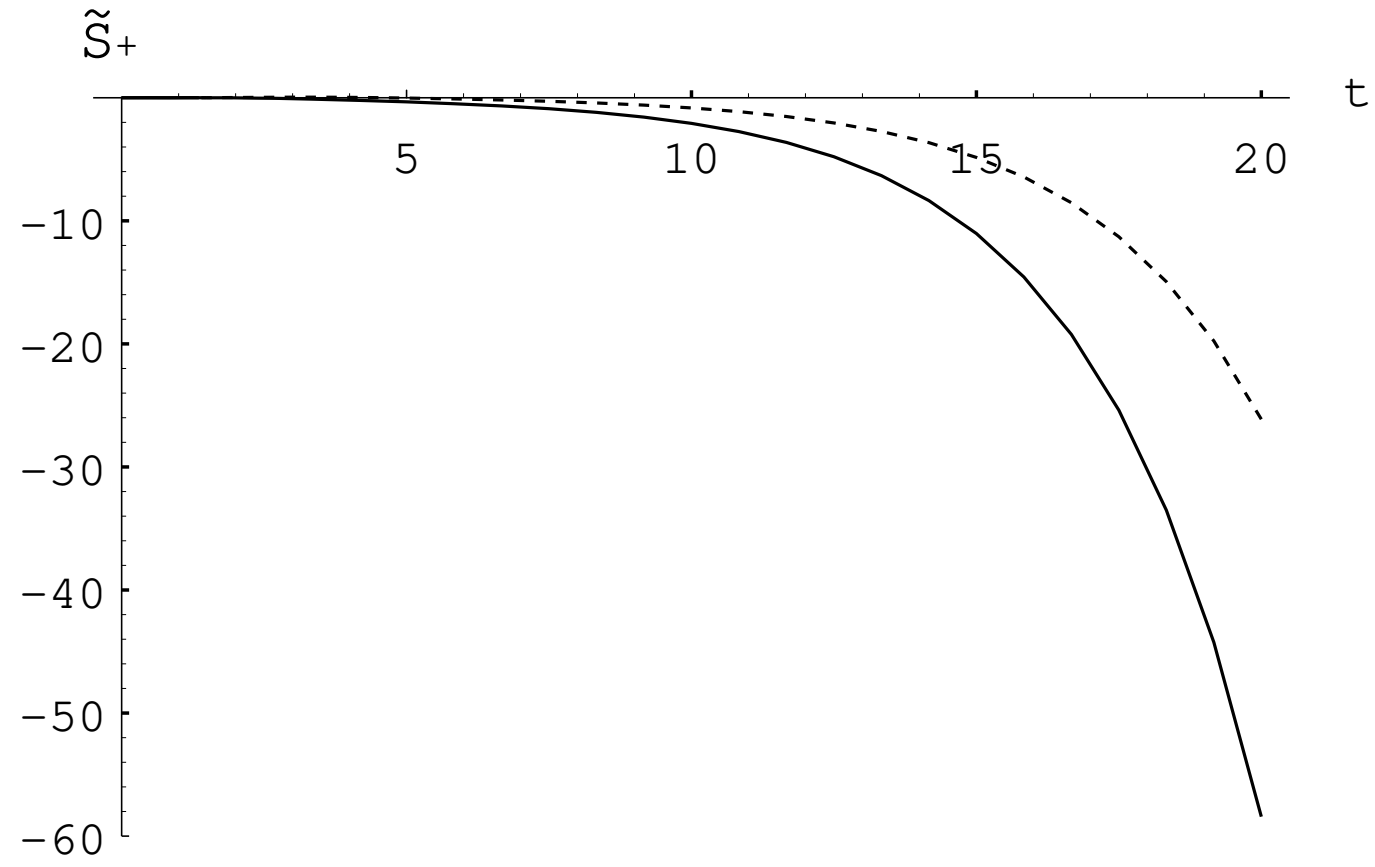

Fig. 3(c)

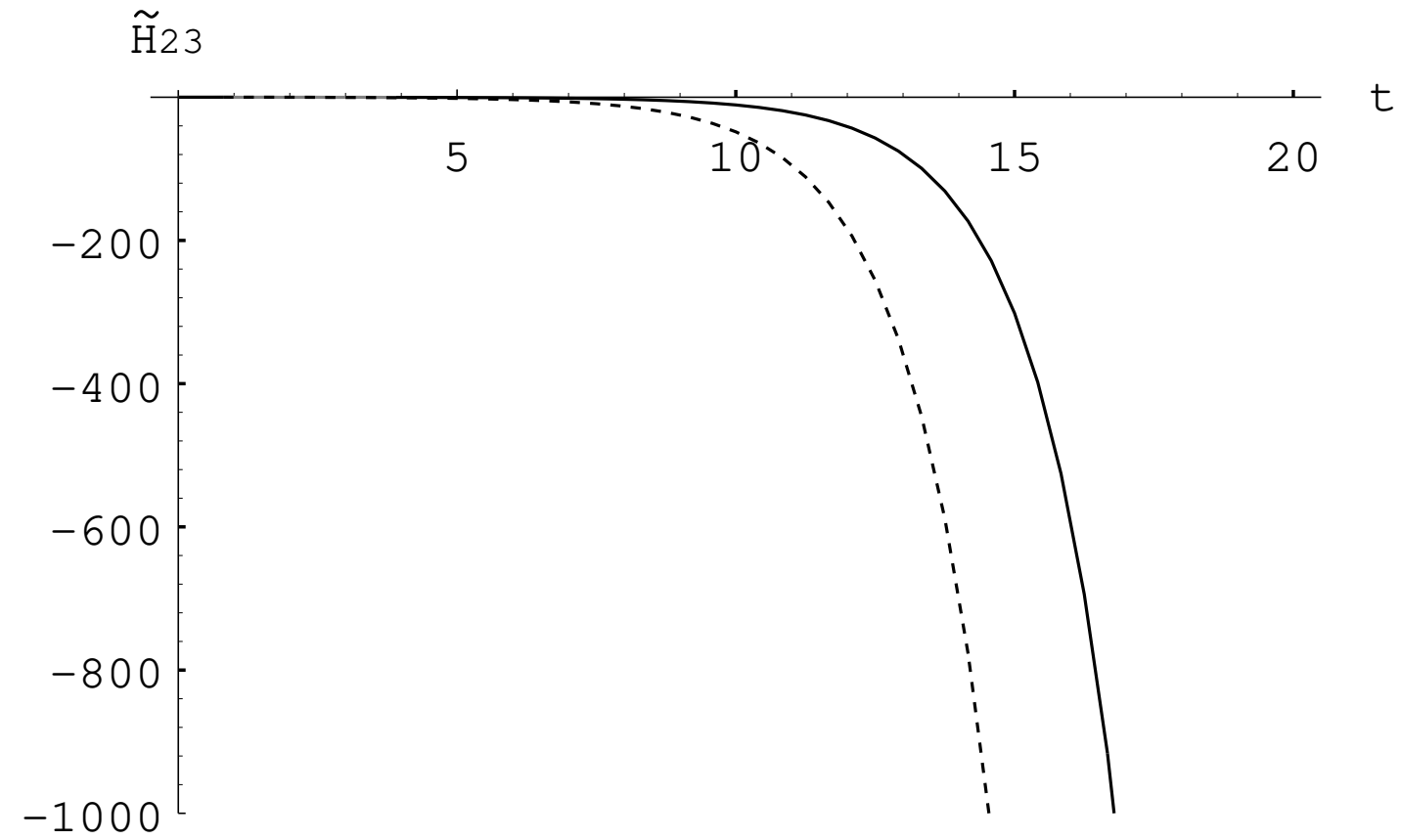

Fig. 3(d) 


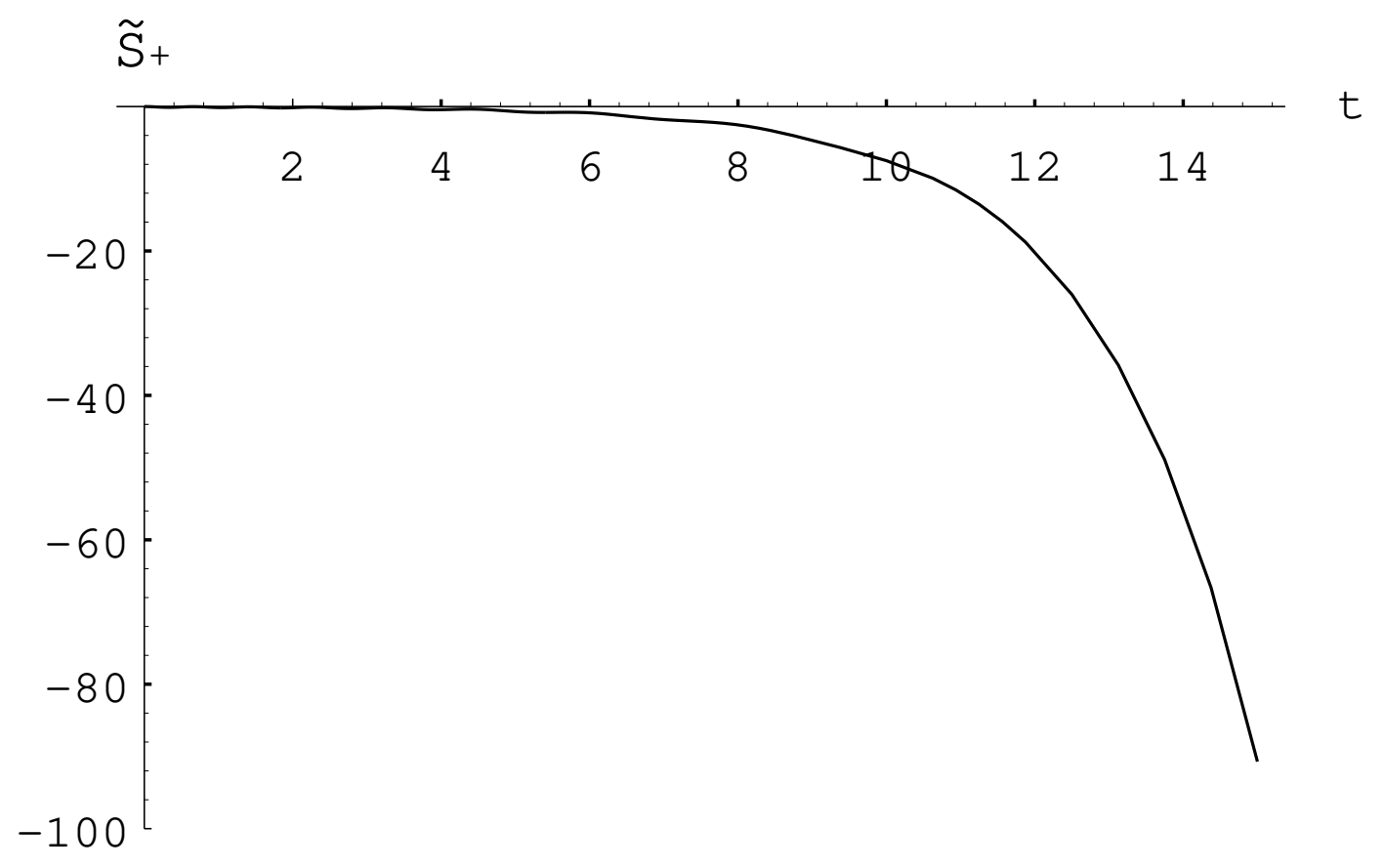

Fig. 3(e)

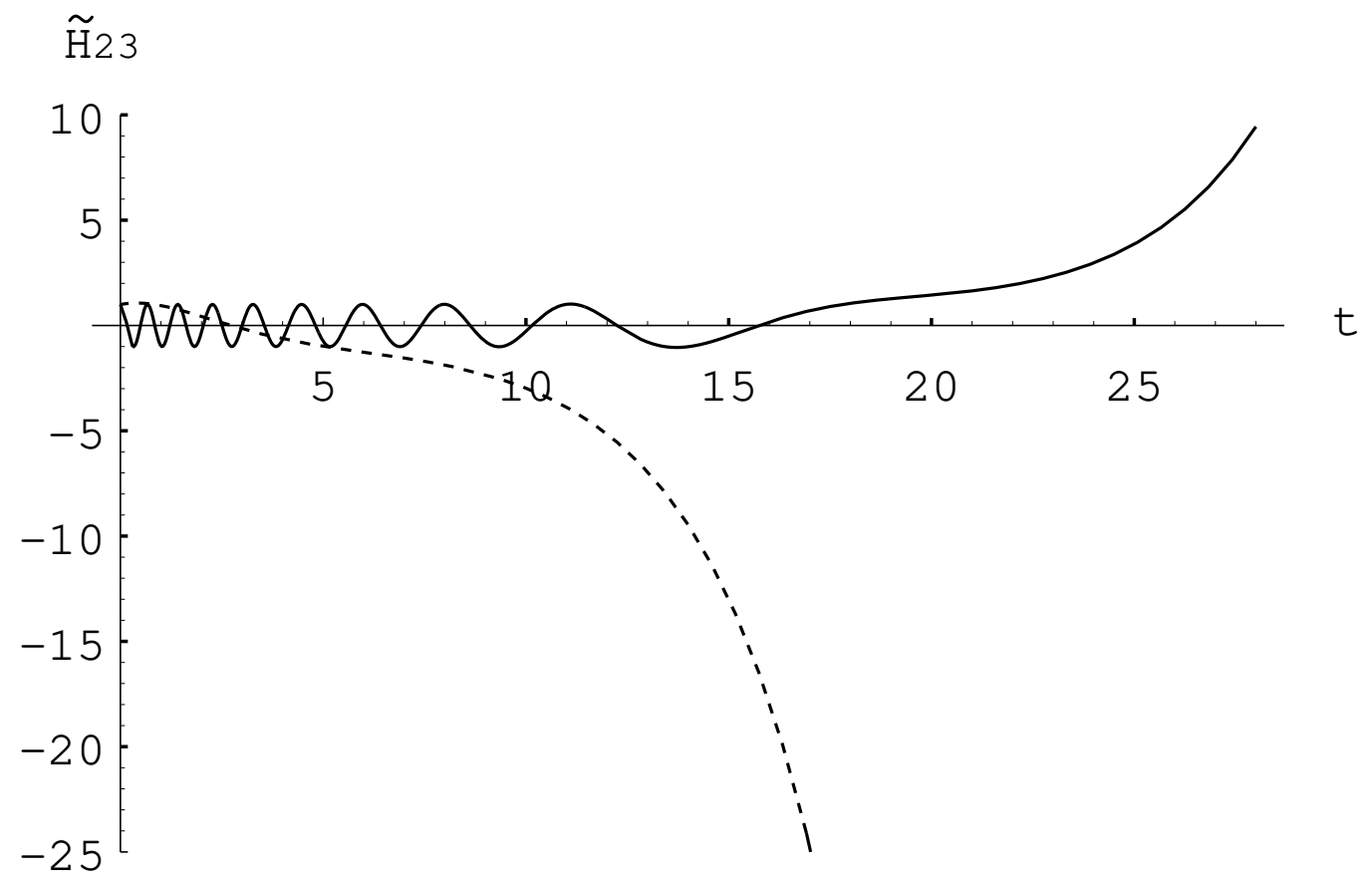

Fig. 3(f) 


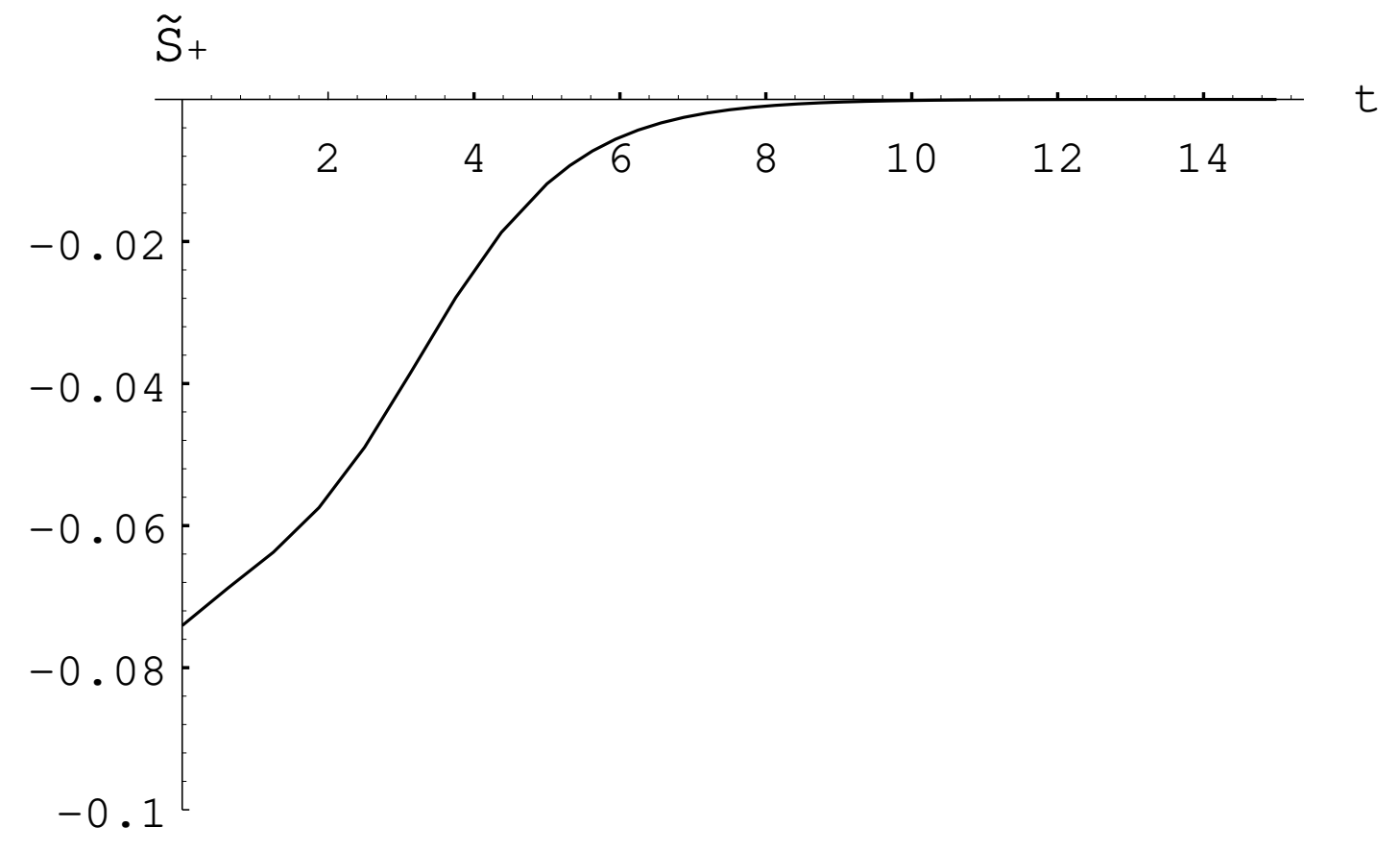

Fig. 4(a)

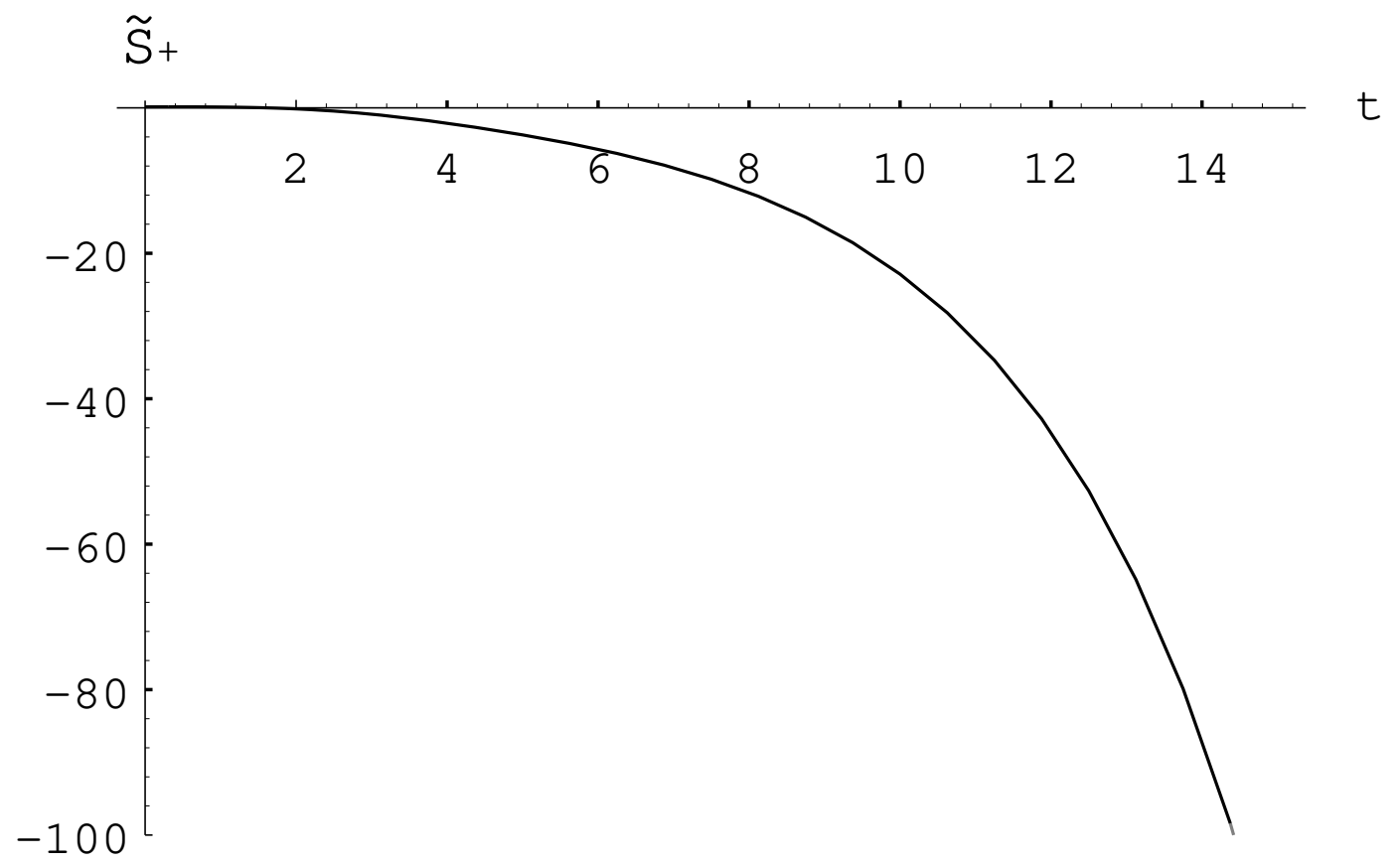

Fig. 4(b) 